\title{
Modeling chemistry during star formation: Water deuteration in dynamic star-forming regions
}

\author{
S. S. Jensen ${ }^{1 \star}$, J. K. Jørgensen ${ }^{1}$, K. Furuya ${ }^{2}$, T. Haugbølle ${ }^{1}$, and Y. Aikawa ${ }^{3}$ \\ 1 Niels Bohr Institute \& Centre for Star and Planet Formation, University of Copenhagen, Øster Voldgade 5-7, DK-1350 Copenhagen \\ K, Denmark \\ 2 National Astronomical Observatory of Japan, Osawa 2-21-1, Mitaka, Tokyo 181-8588, Japan \\ 3 Department of Astronomy, The University of Tokyo, Tokyo, 113-0033, Japan
}

Draft date: March 24, 2021

\begin{abstract}
Context. Recent observations of the $\mathrm{HDO} / \mathrm{H}_{2} \mathrm{O}$ ratio toward protostars in isolated and clustered environments show an apparent dichotomy, where isolated sources show higher $\mathrm{D} / \mathrm{H}$ ratios than clustered counterparts. Establishing which physical and chemical processes create this differentiation can provide new insights into the chemical evolution of water during star formation and the chemical diversity during the star formation process and in young planetary systems.

Aims. We seek to determine to what degree the local cloud environment influences the $\mathrm{D} / \mathrm{H}$ ratio of water in the hot corinos toward low-mass protostars and establish which physical and chemical conditions can reproduce the observed $\mathrm{HDO} / \mathrm{H}_{2} \mathrm{O}$ and $\mathrm{D}_{2} \mathrm{O} / \mathrm{HDO}$ ratios in hot corinos.

Methods. The evolution of water during star formation is modeled using 3D physicochemical models of a dynamic star-forming environment. The physical evolution during the protostellar collapse is described by tracer particles from a 3D MHD simulation of a molecular cloud region. Each particle trajectory is post-processed using RADMC-3D to calculate the temperature and radiation field. The chemical evolution is simulated using a three-phase grain-surface chemistry model and the results are compared with interferometric observations of $\mathrm{H}_{2} \mathrm{O}, \mathrm{HDO}$, and $\mathrm{D}_{2} \mathrm{O}$ in hot corinos toward low-mass protostars.

Results. The physicochemical model reproduces the observed $\mathrm{HDO} / \mathrm{H}_{2} \mathrm{O}$ and $\mathrm{D}_{2} \mathrm{O} / \mathrm{HDO}$ ratios in hot corinos, but shows no correlation with cloud environment when similar initial conditions are tested. The observed dichotomy in water $\mathrm{D} / \mathrm{H}$ ratios requires variation in the initial conditions, for example the duration and temperature of the prestellar phase. Reproducing the observed $\mathrm{D} / \mathrm{H}$ ratios in hot corinos requires a prestellar phase duration $t \sim 1-3 \mathrm{Myr}$ and temperatures in the range $T \sim 10-20 \mathrm{~K}$ prior to collapse. Furthermore, high cosmic-ray ionization rates $\left(\xi_{\mathrm{H} 2} \sim 10^{-15} \mathrm{~s}^{-1}\right)$ appear to be incompatible with the observed $\mathrm{D} / \mathrm{H}$ ratios toward low-mass protostars. Conclusions. This work demonstrates that the observed differentiation between clustered and isolated protostars stems from differences in the molecular cloud or prestellar core conditions and does not arise during the protostellar collapse itself. The observed D/H ratios for water in hot corinos are consistent with chemical inheritance of water, and no resetting during the protostellar collapse, providing a direct link between the prestellar chemistry and the hot corino.
\end{abstract}

Key words. astrochemistry - evolution - radiative transfer - stars: formation - ISM: abundances - methods: numerical

\section{Introduction}

Understanding the formation and evolution of water during star and planet formation is essential to our understanding of the conditions for life in other planetary systems. Water is a prerequisite for life as we know it and, furthermore, also an important molecule for the planet formation process: It contributes significantly to the solid mass reservoirs outside the ice line and impacts the thermal evolution of the gas and the coagulation of dust particles (see, e.g., van Dishoeck et al.|2014).

It is well established that water predominantly forms on dust grain surfaces during the molecular cloud phase, where water constitutes the bulk of the ice (e.g., van Dishoeck et al. 2014). The evolution from the onset of star formation, through the protostellar collapse and protoplanetary disk phases, and finally onto planetary bodies is however still uncertain. Key questions include to what degree water is processed during star and planet formation, namely, whether planets accrete pristine water inherited from the molecular cloud, or if the water is a product of local

\footnotetext{
^ e-mail: sigurd.jensen@nbi.ku.dk
}

processes within the envelope and disk. Crucially, it remains unclear to what extent variations in the local cloud environment impact the water chemistry of the final planetary system, and hence ultimately influence the conditions for biology in extrasolar systems.

Water deuterium fractionation serves as a powerful tracer of the chemical and physical evolution of water during the star and planet formation process, since the degree of deuterium fractionation depends sensitively on the formation environment (e.g., Caselli et al. 2012). Water formed under molecular cloud conditions, where the mean temperature is $T_{\text {gas }} \sim 20 \mathrm{~K}$ and the visual extinction is low ( 1-3 mag), shows a moderate degree of deuterium fractionation, with $\mathrm{D} / \mathrm{H}$ ratios around $10^{-4}-10^{-3}$, compared to the canonical value of $1.5 \times 10^{-5}$ in the local interstellar medium (ISM) (Linsky 2003 1 . Conversely, water formed in prestellar cores, where temperatures are lower $(\sim 10 \mathrm{~K})$ and the visual extinction higher ( $\mathrm{Av}>5 \mathrm{mag}$ ), is highly enriched in deuterium, with $\mathrm{D} / \mathrm{H}$ ratios around $10^{-2}-10^{-1}$. Hence, the $\mathrm{D} / \mathrm{H}$ ratio

\footnotetext{
${ }_{1}$ As either hydrogen atom in $\mathrm{H}_{2} \mathrm{O}$ can be replaced by a deuterium atom, the canonical $\mathrm{HDO} / \mathrm{H}_{2} \mathrm{O}$ ratio is $2 \times(\mathrm{D} / \mathrm{H})_{\text {ism }}=3 \times 10^{-5}$
} 
of water can record information about the formation environment. Deuterium fractionation during star formation is primarily driven by the gas-phase exchange reaction $\mathrm{H}_{3}^{+}+\mathrm{HD} \rightleftharpoons \mathrm{H}_{2} \mathrm{D}^{+}$ $+\mathrm{H}_{2}+\Delta E$, where the exact value of $\Delta E$ depends on the spin state of the involved reactants (Pagani et al. 1992; Hugo et al. 2009). This reaction is exothermic and the backward reaction is inhibited at low temperatures $(T \lesssim 50 \mathrm{~K})$, leading to an enrichment of $\mathrm{H}_{2} \mathrm{D}^{+}$relative to $\mathrm{H}_{3}^{+} \cdot \mathrm{H}_{2} \mathrm{D}^{+}$dissociatively recombines with free electrons to form atomic $\mathrm{D}$, thus increasing the local atomic $\mathrm{D} / \mathrm{H}$ ratio in the gas phase and ultimately on dust grain surfaces where water and other molecules are formed through hydrogenation. Detections of gas-phase $\mathrm{H}_{2} \mathrm{D}^{+}$confirm this enrichment occurs in prestellar cores (Caselli et al. 2003; Vastel et al. 2004).

Observations toward young embedded protostars have revealed a complex evolution of water deuteration during the earliest stages of star formation. Single-dish observations of the cold extended envelope show $\mathrm{HDO} / \mathrm{H}_{2} \mathrm{O}$ ratios of the order of $10^{-2}$ (Parise et al. 2005), while interferometric observations of the hot corino regions show lower ratios in the range $10^{-4}-10^{-3}$ (Coutens et al. 2013, Persson et al. 2014, Jensen et al. 2019). Meanwhile, the gaseous $\mathrm{D}_{2} \mathrm{O} / \mathrm{HDO}$ ratio in hot horinos appear to be of the order of $10^{-2}$ which is an order of magnitude higher than the $\mathrm{HDO} / \mathrm{H}_{2} \mathrm{O}$ ratio in the same region (Coutens et al. 2014). This is schematized in Fig. 1 . A priori, a higher $\mathrm{D}_{2} \mathrm{O} / \mathrm{HDO}$ ratio than $\mathrm{HDO} / \mathrm{H}_{2} \mathrm{O}$ is unexpected: if $\mathrm{H}_{2} \mathrm{O}$, HDO, and $\mathrm{D}_{2} \mathrm{O}$ were formed at the same time through surface reactions the $\left[\mathrm{D}_{2} \mathrm{O} / \mathrm{HDO}\right] /\left[\mathrm{HDO} / \mathrm{H}_{2} \mathrm{O}\right]$ ratio would be the statistical value of 0.25 (Rodgers \& Charnley 2002).

The observed fractionation ratios have been explained by detailed chemical models invoking a layered ice structure with at least two notable components: a water-rich ice mantle with low $\mathrm{D} / \mathrm{H}$ ratio and a surface ice component enriched in deuterium (e.g., Taquet et al. 2013, Furuya et al. 2016, and Fig. 1]. In the cold outer envelope of protostars, the gas-phase $\mathrm{HDO} / \mathrm{H}_{2} \mathrm{O}$ ratio reflects the $\mathrm{D} / \mathrm{H}$ ratio of the surface ice chemistry, namely, water formed during the prestellar core phase with efficient deuterium fractionation, and direct gas phase water formation in this region. Conversely, the $\mathrm{HDO} / \mathrm{H}_{2} \mathrm{O}$ and $\mathrm{D}_{2} \mathrm{O} / \mathrm{HDO}$ ratios in hot corinos reflect the bulk ice reservoir, since the entire ice mantle is thermally desorbed in this region. The complete desorption of the ice layers lowers the $\mathrm{HDO} / \mathrm{H}_{2} \mathrm{O}$ ratio in the gas-phase, since the bulk water ice formed during the molecular cloud phase with a lower degree of deuterium fraction. Meanwhile, the $\mathrm{D}_{2} \mathrm{O} / \mathrm{HDO}$ ratio remains roughly constant, as both $\mathrm{D}_{2} \mathrm{O}$ and $\mathrm{HDO}$ primarily formed during similar physical conditions, in the prestellar core phase. This scenario can explain the observed $\mathrm{D} / \mathrm{H}$ ratios toward young embedded protostars and indicates that the water chemistry observed in hot corinos predominantly originates from the molecular cloud, in other words, an inheritance scenario in which the water is largely inherited from the molecular cloud. Physicochemical models of protoplanetary disks generally support this notion: the $\mathrm{D} / \mathrm{H}$ ratios measured in comets cannot alone be explained by local processing within the disk, and hence some degree of inheritance from the prestellar phase is required to reproduce the high $\mathrm{D} / \mathrm{H}$ ratios of water observed in pristine Solar System material (see, e.g., Cleeves et al. 2014, Furuya et al. 2017; Altwegg et al. 2017).

As the water chemistry appears to be mainly inherited from the environment (i.e., the molecular cloud), the question becomes whether and how the large-scale environment can impact the water chemistry of the final planetary system. In a recent work, evidence for a correlation between the $\mathrm{HDO} / \mathrm{H}_{2} \mathrm{O}$ ratios and the local cloud environment is observed. Jensen et al. (2019) find a higher $\mathrm{HDO} / \mathrm{H}_{2} \mathrm{O}$ ratio toward isolated protostars than what has previously been detected toward sources in clustered environments. The authors proposed two explanations for such a correlation: 1) either temporal differences between isolated and clustered cores: a slower collapse of an isolated prestellar core could prolong the prestellar core phase leading to a higher $\mathrm{D} / \mathrm{H}$ ratio in the water, or 2) higher temperatures or a stronger radiation field in clustered cores, where nearby (proto)stars and turbulent cloud dynamics may heat the gas compared to isolated counterparts. Chemical diversity induced by variations in the natal cloud environment have scarcely been studied theoretically, as the majority of physicochemical models of protostellar collapse have utilized one-dimensional models that fail to capture the complex nature of star formation in dynamic molecular clouds. If local cloud variations drive a difference in the $\mathrm{D} / \mathrm{H}$ ratio of water in the hot corino phase, such a difference may also impact the complex organic molecules (COMs) which are characteristic of hot corinos and hot cores. In a recent study, Aikawa et al. (2020) simulate the impact of various prestellar core conditions on the abundance of COMs and warm carbon chain chemistry (WCCC) in hot corinos. These authors find that the WCCC is more pronounced in cores with lower initial temperature, lower extinction, or a longer prestellar core phase. No clear pattern emerged for the hot corino chemistry; some molecules, such as $\mathrm{CH}_{3} \mathrm{OH}$, are less abundant when the temperature is higher, while other molecules show no impact of variation in prestellar core conditions.

In this work, we combine a detailed 3D MHD model of a star-forming region with radiative transfer and a three-phase chemical model to explore how the local environment may change the chemical evolution through protostellar collapse and determine which conditions can reproduce the observed variation in $\mathrm{HDO} / \mathrm{H}_{2} \mathrm{O}$ ratios toward low-mass protostars. Throughout the paper, we denote $\mathrm{HDO} / \mathrm{H}_{2} \mathrm{O}$ as $f_{\mathrm{D} 1}$ and $\mathrm{D}_{2} \mathrm{O} / \mathrm{HDO}$ as $f_{\mathrm{D} 2}$. Finally, we define the ratio $f_{\mathrm{D} 2} / f_{\mathrm{D} 1}$ as $\alpha$.

\section{Methods}

\subsection{Physical collapse models}

\subsubsection{Molecular cloud models}

A simulation of a $4 \mathrm{pc}^{3}$ region of a molecular cloud is carried out with the adaptive mesh refinement (AMR) code RAMSEs (Teyssier 2002). The entire box is shown in Fig. 2 toward the end of the simulation, where 233 protostars formed in the box. Initial conditions for the molecular cloud, including turbulence driving the star formation process, is identical to the models presented in Haugbølle et al. (2018). The RAMses model presented in this work was modified to include sink particles and tracer particles. Sink particles represent protostars in the model and record the mass accretion onto the protostars. Tracer particles are passive particles injected into the simulation where they are advected along the gas flow, recording the dynamical evolution for chemical post-processing. A detailed description of the model, including these modifications, is available in Haugbølle et al. (2018). The molecular cloud simulation reproduces a number of significant empirical relations for star-forming clouds, such as the Larson scaling relations, the star formation rate (SFR), the core mass function (CMF) and the initial mass function (IMF). The model is therefore well suited to study star formation as a heterogeneous process, where the physical evolution on larger scales may impact the physical and chemical evolution on smaller scales. Compared to Haugbølle et al. (2018), the physical model used in this work has a factor of two higher resolution with a root 


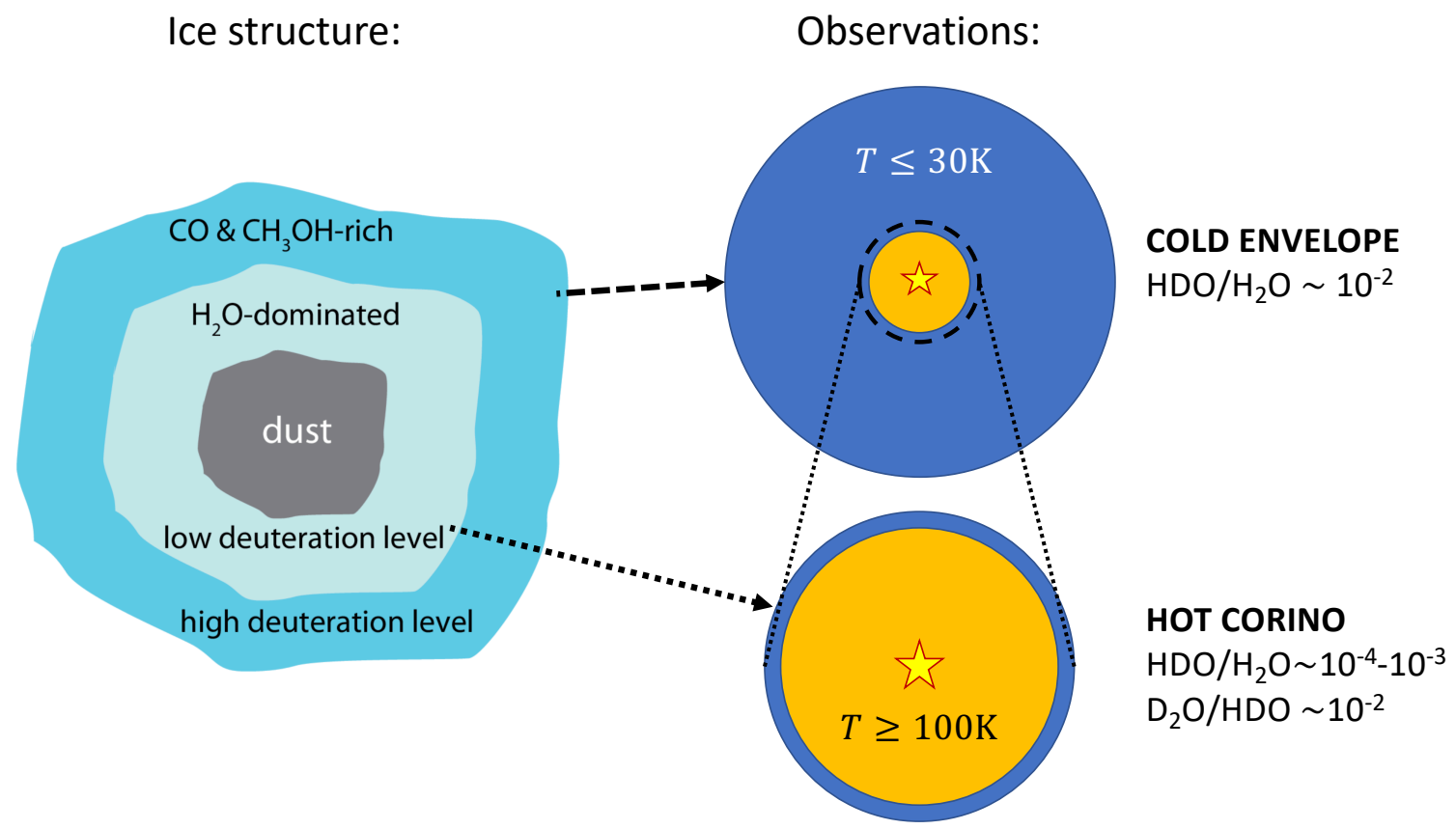

Fig. 1. Schematics of the predicted ice structure in the prestellar core stage (right) and the observed water deuterium fractionation toward embedded low-mass protostars (left). The outer layer of the ice mantle, which has high water D/H ratio, could sublimate in the cold envelope, while the whole mantle is sublimated in the hot corino. The schematical structure is proposed by Furuya et al. (2016). Observational values are from Persson et al. (2014); Coutens et al. (2013, 2014); Jensen et al. (2019).

grid of $512^{3}$. With six levels of refinement this results in a minimum cell-size of 25 au. This resolution is sufficient to study the dynamics from the larger molecular cloud scales down to the collapse of individual protostellar cores, but is not suitable to resolve any emerging accretion disk. The accretion rate onto sink particles within the simulation was recorded at a cadence of $\sim 100$ years, while the physical structure of the simulation was recorded in 'snapshots' at a lower cadence, once every $5 \mathrm{kyr}$. We calculated the positions of the tracer particles between each snapshot from the position, velocity, and acceleration vectors at each snapshot. For each sink particle, we followed tracer particles for a duration of $\sim 350 \mathrm{kyr}$, starting with a pre-collapse phase $100 \mathrm{kyr}$ before the formation of the sink particle (i.e., the onset of protostellar collapse) and continuing through the Class 0 and Class I phases. Including a longer pre-collapse phase was not possible, as several of the studied sink particles form early in the simulation. An overview of the different phases of the simulation is shown in Fig. 3 During the pre-collapse phase, we averaged the density in $10 \mathrm{kyr}$ windows to reduce computational time in this phase, where the temperature remains fixed.

As no disks form within the simulation, the evolution toward the end of the simulated time range is less accurate as the formation of an accretion disk changes the dynamics and radiation field in the inner part of the core. We note that higher-resolution simulations performed within the same framework, such as the models presented in Kuffmeier et al. (2018), show that accretion disks form when the resolution is increased. We focus on the earlier stages of star formation in this work, namely the protostellar
Table 1. Overview of the simulated protostars studied in this work. Isolated and clustered sources are denoted I and C, respectively. The envelope mass, $M_{\mathrm{env}}$, is the total mass within a 10,000 au sphere at the onset of collapse (i.e., at the moment the sink particle is formed). The free-fall timescale is calculated assuming a uniform density within 10,000 au. The quantity $M_{\text {final }}$ indicates the final mass at the end of the simulation.

\begin{tabular}{lllll}
\hline \hline Sink & $M_{\text {final }}\left(\mathrm{M}_{\odot}\right)$ & $M_{\text {env }}\left(\mathrm{M}_{\odot}\right)$ & $t_{\text {ff }}(\mathrm{kyr})$ & $\mathrm{I} / \mathrm{C}$ \\
\hline M022-C & 0.22 & 1.50 & 144.5 & $\mathrm{C}$ \\
M023-I & 0.23 & 0.90 & 186.4 & $\mathrm{I}$ \\
M048-C & 0.48 & 1.06 & 171.6 & $\mathrm{C}$ \\
M048-I & 0.48 & 1.19 & 162.2 & $\mathrm{I}$ \\
M049-C & 0.49 & 0.73 & 206.5 & $\mathrm{C}$ \\
M072-I & 0.72 & 0.85 & 192.2 & $\mathrm{I}$ \\
M072-C & 0.72 & 5.58 & 74.8 & $\mathrm{C}$ \\
M073-C & 0.73 & 0.94 & 182.5 & $\mathrm{C}$ \\
M101-C & 1.01 & 1.74 & 134.1 & $\mathrm{C}$ \\
\hline
\end{tabular}

collapse down to hot corino scales $(\sim 100 \mathrm{au})$. A sample of lowmass protostars, both clustered and isolated, were selected from the simulation. Throughout this work, we define isolated protostars as sink particles where no other protostars enter within 20,000 au during the simulation. For the clustered sources, we require at least two neighboring protostars within 10,000 au at some point in the simulated evolution. The characteristics of the selected sample is summarized in Table 1 . 


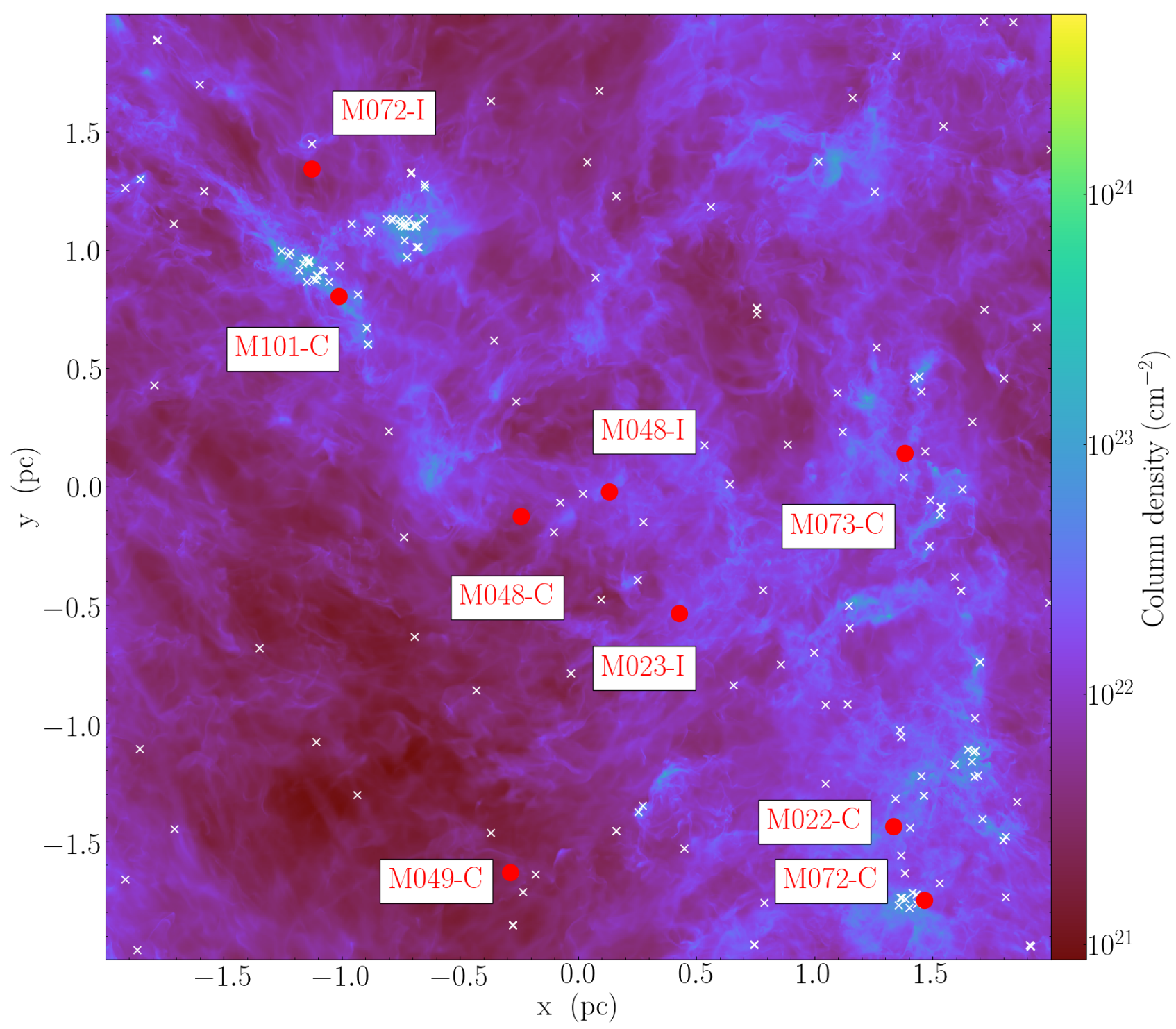

Fig. 2. Projected densities in the molecular cloud simulation. A total of 233 protostars, denoted with white crosses, form by the end of the simulation $(\mathrm{t}=1.65 \mathrm{Myr})$. Most protostars form in clusters along filaments. The protostars studied in this work are denoted with red circles.

\subsubsection{Radiative transfer models}

The dust temperatures and radiation field around each sink particle was post-processed using the Monte Carlo radiative transfer code RADMC-3D (Dullemond et al. 2012). For each sink particle in the RAMSES simulation, a spherical cutout of 30,000 au around the sink particle was converted to a RADMC-3D AMR grid. Creating smaller cutouts of the total grid is necessary to reduce the memory load, which can be substantial for large photon packages. The dust temperature is calculated via the MCTHERM routine; we injected 15 million photons in each radiative transfer model to sample the grid adequately. Models with a higher number of photons were tested and the results of the radiative transfer converge to the same result. We utilized the dust opacities from Semenov et al. (2003) in the radiative transfer modeling.

The protostars, represented by the sink particles, are included in the radiative transfer models as point sources. In addition to the central protostar in each cutout, we included neighboring protostars entering within 10,000 au of the protostar in focus.
The protostellar luminosity is interpolated from the pre-main sequence (PMS) models from D'Antona \& Mazzitelli (1997). We included a $100 \mathrm{kyr}$ offset between the onset of core collapse and the PMS age, similar to Young \& Evans (2005), corresponding to approximately one free-fall timescale. In the first $100 \mathrm{kyr}$ of the collapse, the surface temperature of the protostar is fixed at $2000 \mathrm{~K}$. The radius is set to be $2.5 R_{\odot}$ for the calculation of the accretion luminosity. This implementation is similar to that presented in Frimann et al. (2016).

In addition to the protostellar luminosity derived from the PMS models, we included the accretion luminosity of the central protostar from Eq. 1 .

$L_{\mathrm{acc}}=G \frac{M \dot{M}}{2 R}$.

The accretion rates onto the sink particles were recorded with a higher cadence than the RAMSES output. To account for this difference in sampling, we ran a total of five RADMC-3D models for each 


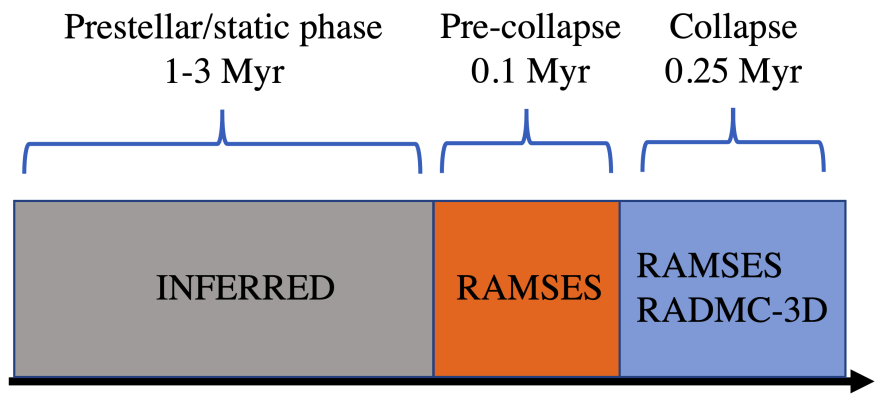

Time

Fig. 3. Overview of the different phases of the model. In the initial prestellar (static) phase, the physical conditions are inferred previous simulations and observations (e.g., Keto \& Caselli 2008). In the precollapse phase, which has a fixed duration of $100 \mathrm{kyr}$, the physical evolution is derived from RAMSEs, but since the protostar is not yet formed, no radiative transfer is performed. During the collapse, the physical evolution is derived from RAMSES and post-processed using RADMC-3D. The duration of the collapse varies between individual tracer particles, with an upper duration of $\sim 250 \mathrm{kyr}$.

RAMSES output with varying accretion luminosities: we created a linear grid of four models, ranging from the lowest accretion rate to the highest accretion rate associated with any given RAMSEs output. We calculated the fifth model using the mean accretion rate during the period between the snapshots. When estimating the dust temperature for a tracer particle at a given position and time, we calculated the total luminosity $\left(L_{\mathrm{acc}}+L_{\text {protostar }}\right)$ of the protostar at that time, and interpolated the temperature from the values provided by the five RADMC-3D models. We assume that the dust and gas is thermally coupled, such that the gas temperatures and dust temperatures are equal. This approach is reasonable at higher densities present during the protostellar collapse ( $n \gtrsim 10^{4}$, Goldsmith 2001), but may not be valid in the prestellar phase where densities are lower. The limitations of the model are discussed in Sect.4.2. A lower limit on the temperature of $10 \mathrm{~K}$ is set throughout the chemical modeling. While lower temperatures can occur in the center of dense cores, this is a reasonably average temperature for dense cores (e.g., Pagani et al. 2007). Furthermore, many reactions in the chemical network are validated to a lower limit of $10 \mathrm{~K}$.

Along with the thermal dust calculation, the mean UV radiation field is calculated in the RADMC-3D grid with the MCMONO routine. In addition to the protostellar sources, the models include an external radiation field in the form of the interstellar radiation field (ISRF) from Draine (1978), denoted as $G_{0}$, which irradiates the 30,000 au RADMC-3D domain around each sink particle. For the MCMONo calculations, we opted to run just one model using the mean accretion luminosity for each RAMSES snapshot. As the UV flux emerging from the central protostar is heavily attenuated, the calculated UV field is dominated by the external radiation field, except for the innermost 25-50 au around a protostar. Running five models with varying accretion luminosity would hence not have a notable impact on the surrounding envelope and the chemical evolution herein. Figure 4 shows an example of the thermal structure calculated around one of the sinks studied in this work.

We note that the earliest protostellar evolution may not be well explained by the PMS models, as highlighted by the luminosity problem (see, e.g., Jensen \& Haugbølle 2018, and references therein). We do not consider this a major issue for this work for two reason. First, the accretion luminosity, not the pro- tostellar luminosity, is the primary luminosity source at the very early stages modeled here. Second, the purpose of this work is to study how variations in environment influences the chemistry during star formation and does not depend on the exact choice of PMS model. Additionally, we note that the PMS phase occurs toward the end of the range of the time-domain studied in this work, namely, the $\sim 250 \mathrm{kyr}$ of the protostellar collapse after the initial collapse of the core.

\subsection{Calculating extinction in the cores}

For photodissociation and photodesorption, rate coefficients are given as a function of the visual extinction $A_{\mathrm{v}}$. From the calculated mean field intensities, we follow the approach of Visser et al. (2011) and derive the optical depth $\tau_{U V}$ at UV wavelengths from:

$\tau_{\mathrm{UV}}=-\ln \frac{F_{\mathrm{UV}, \mathrm{RADMC}-3 \mathrm{D}}}{F_{\mathrm{UV}, 0}}$,

where $F_{\mathrm{UV}, \mathrm{RADMC}-3 \mathrm{D}}$ is the integrated UV flux from the radiative transfer calculation, and $F_{\mathrm{UV}, 0}$ is either the integrated UV flux from the ISRF or from the central protostar, whichever is higher in each grid point. Initially, the UV flux from the protostar is quite low owing to the low surface temperature of the protostar $(2000 \mathrm{~K})$ and during this period, the ISRF dominates the UV flux. Later on, the UV flux from the protostar may increase, however, the flux is not able to penetrate the envelope, because no outflow cavities are resolved in the simulation.

From the optical depth, the visual extinction is calculated as $A_{\mathrm{v}}^{\mathrm{RADMC}-3 \mathrm{D}}=\tau_{\mathrm{UV}} / 3.02$. In addition to the extinction calculated with RADMC-3D, we assume an ambient extinction everywhere in the molecular cloud, (i.e., surrounding the 30,000 au radiative transfer domain). The total extinction during the collapse is then given as $A_{\mathrm{v}}=A_{\mathrm{v}}^{\text {cloud }}+A_{\mathrm{v}}^{\mathrm{RADMC}-3 \mathrm{D}}$, where we assume $A_{\mathrm{v}}^{\text {cloud }}=5 \mathrm{mag}$ in our fiducial model. Models with a lower ambient extinction, $A_{\mathrm{v}}^{\text {cloud }}=2 \mathrm{mag}$, are presented in appendix $\mathrm{D}$. The column densities are estimated using the empirical relation $N_{\mathrm{H}}=A_{\mathrm{V}} \times 1.59 \times 10^{21} \mathrm{~cm}^{-2}$ (Bohlin et al. 1978; Diplas \& Savage 1994). These parameters are necessary to derive the self-shielding functions and photochemical rates for the chemical models.

As mentioned in the previous section, each tracer particle trajectory includes $100 \mathrm{kyr}$ of evolution prior to the formation of the sink particle. Since the protostar is not formed in the precollapse phase, no radiative transfer is calculated, and we use a fixed temperature of $10 \mathrm{~K}$ and a fixed visual extinction of $A_{\mathrm{v}}=$ 5 mag during this period.

\subsection{Chemical model}

The chemical evolution was simulated using the rate-equation approach (see, e.g., Cuppen et al. 2017). The model adopts the three-phase model introduced by Hasegawa \& Herbst (1993), with a number of modifications detailed below. Three-phase models consider the ice mantle and ice surface as distinct phases, as opposed to two-phase models where the ice species all reside in the same (bulk) phase (Hasegawa et al. 1992). As the chemistry evolves, two-phase models may produce inaccurate results, since these models cannot distinguish between species present in the reactive surface layers and species buried deep in the ice mantle where the molecules are inert or less likely to react. Three-phase models overcome some of these issues by separating ice species in surface and mantle phases, however other 


\section{sink M048-C}

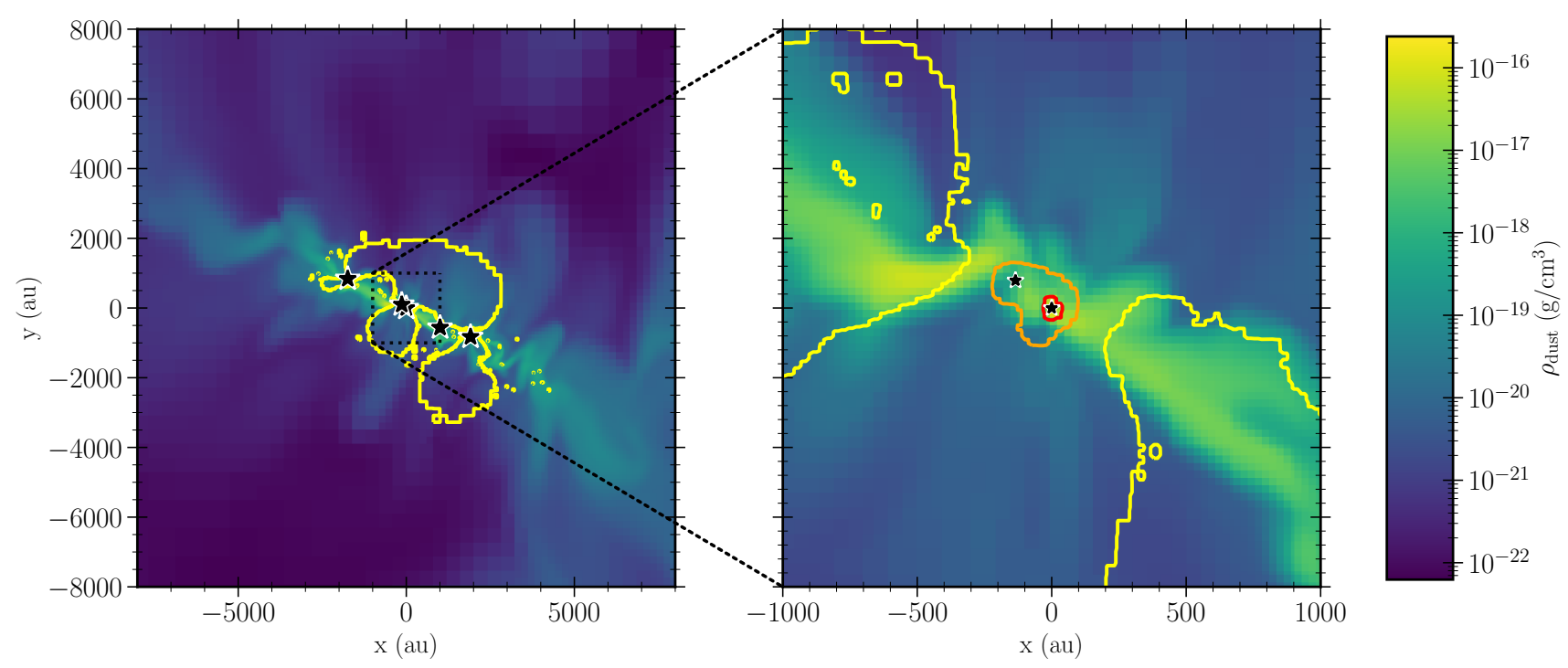

Fig. 4. Structure around the binary sink M048-C with a final mass of $M=0.48 \mathrm{M}_{\odot}$. The color map shows the density and reveals that the sink, along with several siblings, is born along a filament, extending from northwest to southeast in the imaged plane. Contours show the temperature, calculated via RADMC-3D, in levels of [25.0, 50.0, 100.0] K. In the right panel, the yellow $25 \mathrm{~K}$ contour is incomplete; the left panel shows the full structure of this region. The image shows a slice through the grid, not a projection.

caveats are introduced with these models, which we will discuss later. We do not consider bulk diffusion in our model; the effectiveness of bulk reactivity is still a matter of debate (see, e.g., Shingledecker et al. 2019).

Experiments have shown that surface chemistry likely occurs in the top two to four monolayers ${ }^{2}$. Motivated by these results, we adopted a similar approach to that of Furuya et al. (2015), in which we extended the surface layer to thickness of four monolayers. Other three-phase models, such as the NAUTILUs, model have adopted a similar approach, albeit with two monolayers instead of four (Ruaud et al. 2016). Henceforth, we refer to the surface phase of the model as the surface layer, although the thickness of the phase is four monolayers.

\subsubsection{Chemical network}

For the gas-phase chemistry, we adopted the chemical network presented by Majumdar et al. (2017), which extends the KIDA ${ }^{3}$ network to include spin-state chemistry of $\mathrm{H}_{2}, \mathrm{H}_{3}^{+}$and other key molecules necessary to accurately model deuterium fractionation processes in the cold ISM. The basis of the spin-state chemistry was presented in Sipilä et al. (2015). The gas-phase network was initially benchmarked in Majumdar et al. (2017) for dark cloud conditions and found to agree with observations. In this work, the network was updated to include more recent photodissociation rates from the Leiden photodissociation database (Heays et al. 2017), a different treatment of grain recombination reaction, and other minor adjustments. We refer to the papers of Wakelam et al. (2015) and Majumdar et al. (2017) for a detailed description of the network and reactions. We excluded three-body reactions from these calculations, as they are inefficient under typical ISM conditions (Cuppen et al.2017).

\footnotetext{
2 One monolayer is defined as the amount of binding sites on the grain surface, such that one monolayer corresponds to every site on the dust grain being occupied

3 http://kida.astrophy.u-bordeaux.fr
}

For the surface network, we used a network derived from Taquet et al. (2013), which was further extended to include reactions from Garrod (2013). The network has been extended to include single and doubly deuterated isotopologues for molecules with up to four carbon atoms. For a number of key species such as methanol, we included all deuterated isotopologues, such as $\mathrm{CD}_{3} \mathrm{OD}$.

Binding energies from Garrod \& Herbst (2006) were used for the majority of the species, with updated estimates from Wakelam et al. (2017) used where applicable. For deuterated isotopologs, we used the same binding energy as the main species, except for atomic $\mathrm{D}$, which is set $21 \mathrm{~K}$ higher than $\mathrm{H}$ atoms (Caselli et al.2002). For the extensive network used in this work, a few species do not have any binding energy estimates. For these, we use the binding energy of $\mathrm{H}_{2} \mathrm{O}$ as a "default" value.

The full chemical network and the chemical code is publicly available for reference and provided for the community for future work 4

\subsubsection{Adsorption and desorption}

For the adsorption onto the grain surface and thermal desorption of surface species, we followed the method of Hasegawa et al. (1992). The adsorption rate coefficient is given by:

$k_{\text {ads }}(i)=S\langle v(i)\rangle \sigma_{\text {dust }} n_{\text {dust }} \quad\left(\mathrm{s}^{-1}\right)$,

where $\sigma_{\text {dust }}=\pi r_{\text {dust }}^{2}$ is the cross-section of dust grains, $n_{\text {dust }}$ is the number density of dust grains, and $\langle v(i)\rangle=\sqrt{8 k_{\mathrm{b}} T / \pi m_{i}}$ is the mean thermal velocity of the species $i$. There parameter $S$ is the sticking coefficient, which is estimated from the experimentally derived prescription presented by He et al. (2016).

The thermal desorption is calculated from the first-order expression as follows:

$k_{\mathrm{des}}(i)=v_{i} \exp \left(\frac{-E_{\text {bind }}}{k_{\mathrm{b}} T}\right) \quad\left(\mathrm{s}^{-1}\right)$,

4 https://github.com/ssjensen92/kemimo

Article number, page 6 of 23 
where $E_{\text {bind }}$ is the binding energy of the species on the surface. $v_{i}=\sqrt{\left(2 N_{\mathrm{s}} E_{\mathrm{bind}}\right) /\left(\pi^{2} m_{i}\right)}$ is the characteristic frequency for the species, calculated assuming a harmonic potential for the surface binding sites (Tielens \& Allamandola 1987). The quantity $N_{\mathrm{s}}$ is the number of binding sites per dust grain and $m_{i}$ is the mass of the species.

Over time, incident cosmic-ray particles heat dust grains (e.g., Hasegawa \& Herbst 1993). This leads to a cosmic-ray induced desorption rate, which we calculated following Leger et al. (1985):

$k_{\mathrm{des}, \mathrm{CR}-\text { induced }}=f(70 \mathrm{~K}) k_{\mathrm{des}}(70 \mathrm{~K}) \quad\left(\mathrm{s}^{-1}\right)$,

where the factor $f$ determines the fraction of time the dust grains spend at $70 \mathrm{~K}$. This value is determined through the balance between heating rate of dust grains by cosmic-ray particles and the cooling rate through radiation as follows: $f(70 K)=$ $\frac{\zeta_{C} R}{1.3 \times 10^{-17}} \times 3.16 \times 10^{-19}$.

\subsubsection{Surface reactions}

We modeled surface chemistry reactions using the LangmuirHinshelwood mechanism, which assumes that both reactants adsorb onto the grain surface before any reaction occurs (Cuppen et al. 2017). After adsorption, the reactants diffuse thermally across the grain surface and upon encounter they may react, depending on the reaction probability. The rate coefficient $k_{i j}$ for this mechanism is given as

$k_{\mathrm{LH}}(i, j)=\kappa_{i j}\left(k_{\mathrm{hop}}(i)+k_{\mathrm{hop}}(j)\right) \frac{1}{N_{\text {layer }} N_{\mathrm{s}} n_{\text {dust }}} \quad\left(\mathrm{cm}^{3} \mathrm{~s}^{-1}\right)$,

where $N_{\text {layer }}=n_{\mathrm{s}} /\left(n_{\text {dust }} n_{\mathrm{s}}\right)$ is the current number of surface layers in monolayers. In this equation, $n_{\mathrm{s}}$ denotes the sum of the number densities of all surface species, $n_{\text {dust }}$ denotes the number density of dust grains, $\kappa_{i j}$ is the reaction probability, and $k_{\text {hop }}$ is the thermal hopping rate given by $k_{\text {hop }}=v_{i} \exp \left(-E_{\text {diff }} / k_{\mathrm{b}} T\right)$. We assume $E_{\text {diff }}=0.5 \times E_{\text {bind }}$ in this work. The reaction probability $\kappa_{i j}$ is unity for barrierless reaction. For reactions with barriers, the barrier is overcome either through quantum tunneling or thermal hopping, whichever is the fastest. We included reactiondiffusion competition as introduced by Garrod \& Pauly (2011). This mechanism considers that two reactants encountering oneanother in a binding site may have multiple chances to react before one of them diffuses out of the site, hence increasing the reaction probability of reactions with barriers. The inclusion of reaction-diffusion competition is pronounced. For instance, the primary formation pathway of $\mathrm{H}_{2} \mathrm{O}$ changes from the barrierless reaction $\mathrm{H}+\mathrm{OH} \rightarrow \mathrm{H}_{2} \mathrm{O}$ to the reaction $\mathrm{H}_{2}+\mathrm{OH} \rightarrow \mathrm{H}_{2} \mathrm{O}+\mathrm{H}$, which has a barrier of $2100 \mathrm{~K}$, when the mechanism is included (Furuya et al. 2015). The reaction probability when assuming reaction-diffusion competition is calculated from:

$\kappa_{i j}=\frac{\kappa_{i j}^{0}}{\kappa_{i j}^{0}+k_{\mathrm{hop}}(i)+k_{\mathrm{hop}}(j)}$,

where $\kappa_{i j}^{0}$ is the probability to overcome the reaction barrier. The probability of crossing an activation barrier $E_{\mathrm{A}}$ through thermal hopping is given by $\kappa_{i j}^{0}=\exp \left(-E_{\mathrm{A}} / k_{\mathrm{b}} T\right)$, while the expression for quantum tunneling depends on the choice of potential barrier. For a rectangular barrier of width $a$, the expression is $\kappa_{i j}^{0}=\exp \left[-2(a / \hbar)\left(2 \mu E_{\mathrm{A}}\right)^{1 / 2}\right]$. In this expression, $\mu$ denotes the reduced mass of the reactants. We adopted a rectangular barrier with a width of $1 \AA$, except for a number of reactions listed in appendix A

For exothermic reactions, products may desorp from the grain surface owing to the excess energy released during the reaction. This process is known as chemical desorption and efficiency of this process is poorly constrained, but could play an important role for the interplay between gas-phase and grainsurface chemistry in star-forming regions. We included chemical desorption using the prescription of Garrod et al. (2007) for the probability of desorption for reactions with one product, with an efficiency parameter of $a=0.01$ (see, e.g., Cuppen et al.2017). Reactions with multiple products are not allowed to chemically desorp.

\subsubsection{Photochemistry}

The chemical model includes photodissociation and photodesorption of species in the surface layer. Data on photodissociation cross-sections for surface species are sparse and we adopted a similar approach as in Furuya et al. (2015) for the surface photochemistry. In this approach, gas-phase photodissociation rates are rescaled for the corresponding surface reaction. The scaling factor is determined from the ratio of the gas-phase to surface rates for water: $k_{i j}^{\text {surface }}=k_{i j}^{\text {gas }} \times k_{\mathrm{H}_{2} \mathrm{O}}^{\text {surface }} / k_{\mathrm{H}_{2} \mathrm{O}}^{\text {gas }}$. The rate coefficient for the photodissociation is given as:

$k_{\text {photodiss }}(i)=\theta_{i} P_{\mathrm{abs}}(i) \sigma_{\mathrm{dust}} n_{\mathrm{dust}} F_{\mathrm{UV}}(i) \times \min \left(N_{\text {layer }}, 4\right) \quad\left(\mathrm{s}^{-1}\right)$,

where $\theta_{i}$ is the surface coverage factor of the species, $\theta_{i}=$ $n(i) / n_{\mathrm{s}}, F_{\mathrm{UV}}$ is the UV flux in photons $\mathrm{cm}^{-2} \mathrm{~s}^{-1}$, and we allowed photodissociation in the top four monolayers, that is, the surface layer in the three-phase model. In our model, it is implicitly assumed that the photo-fragments in the ice mantle recombine immediately. While this may lead to an increase in the abundance of radicals and trigger the formation of COMs, the effect on the water chemistry studied in this work is expected to be limited as a result of the high extinction in these environments. The photodesorption rates are given by

$k_{\text {photodes }}(i)=Y_{i} \theta_{i} \sigma_{\text {dust }} n_{\text {dust }} F_{\mathrm{UV}}(i) \times \min \left(N_{\text {layer }} / 4,1\right) \quad\left(\mathrm{s}^{-1}\right)$,

where $Y_{i}$ is the effective yield per photon for thick ice. For photodesorption, we likewise allowed reactions in up to four monolayers. Photodesorption yields for $\mathrm{CO}, \mathrm{CO}_{2}$, and $\mathrm{N}_{2}$ are derived from experiments (Oberg et al. 2007, Öberg et al. 2009, Fayolle et al. 2011, 2013). The CO yield for thick ice is $2.7 \times 10^{-3}$, while for $\mathrm{CO}_{2}$ the total yield is $10^{-3}$, with an equal branching ratio between the outcomes $\mathrm{CO}^{\text {gas }}+\mathrm{O}^{\text {gas }}$ and $\mathrm{CO}_{2}^{\text {gas }}$. The desorption yield for $\mathrm{N}_{2}$ is set to $5 \times 10^{-4}$. For $\mathrm{H}_{2} \mathrm{O}, \mathrm{HDO}$, and $\mathrm{D}_{2} \mathrm{O}$, we adopted the branching ratios for photodissociation and photodesorption presented by Arasa et al. (2015). The absorption probability for $\mathrm{H}_{2} \mathrm{O}$ per incident UV photon is set to $5 \times 10^{-3}$. For the remaining species we used a generic yield of $10^{-3}$. While the network was developed to include complete photochemistry, we note that in the models presented in this work the photon flux is heavily attenuated for the majority of the time, and therefore the effect of photochemistry is limited. The model included self-shielding for $\mathrm{H}_{2}, \mathrm{HD}, \mathrm{CO}$, and $\mathrm{N}_{2}$. For $\mathrm{CO}$ and $\mathrm{N}_{2}$, we interpolated the shielding factor from the tables of Visser et al. (2009) and Heays et al. (2014). For $\mathrm{H}_{2}$ and HD we used the parametric approximations derived by Draine \& Bertoldi (1996); Richings et al. (2014) and Wolcott-Green \& Haiman (2011), respectively. 


\subsection{Benchmarking the network}

The chemical model was benchmarked against the gas-grain model presented in Furuya et al. (2015) and Furuya et al. (2016), which successfully reproduced the observed $\mathrm{HDO} / \mathrm{H}_{2} \mathrm{O}$ and $\mathrm{D}_{2} \mathrm{O} / \mathrm{HDO}$ ratios toward protostellar cores. We refer to this model as the reference model throughout this section. Initially, a 10 Myr steady-state simulation with $n_{\mathrm{H}}=2 \times 10^{4} \mathrm{~cm}^{-3}$, $T=10 \mathrm{~K}$, and $A_{\mathrm{v}}=5 \mathrm{mag}$ was compared, shown in Fig. 5 The cosmic-ray ionization rate for $\mathrm{H}_{2}$ is set to $\xi_{\mathrm{H}_{2}}=1.3 \times 10^{-1 /} \mathrm{s}^{-1}$. The abundances of major species, such as $\mathrm{H}_{2} \mathrm{O}, \mathrm{CH}_{3} \mathrm{OH}$, and $\mathrm{CO}_{2}$ show good agreement, while the $\mathrm{CO}$ abundance differ by a factor of $\sim 2$. This is due to the inclusion of the hydrogen abstraction reaction:

$\mathrm{HCO}+\mathrm{H} \rightarrow \mathrm{CO}+\mathrm{H}_{2}$

This reaction is not included in the reference model, but was recently demonstrated as a pathway for $\mathrm{CO}$ formation (Chuang et al. 2016). As in the reference model, $\mathrm{H}_{2} \mathrm{O}$ and $\mathrm{HDO}$ are primarily formed in the surface chemistry, while $\mathrm{D}_{2} \mathrm{O}$ is dominantly formed in the gas phase. The principal formation pathway of $\mathrm{H}_{2} \mathrm{O}$ is the surface reaction $\mathrm{H}_{2}+\mathrm{OH} \rightarrow \mathrm{H}_{2} \mathrm{O}+\mathrm{H}$, with a barrier of $2100 \mathrm{~K}$. This pathway dominates when reaction-diffusion competition is included in the model. Without reaction-diffusion competition, the dominant pathway to $\mathrm{H}_{2} \mathrm{O}$ formation would be the surface reaction $\mathrm{OH}+\mathrm{H} \rightarrow \mathrm{H}_{2} \mathrm{O}$, as in Furuya et al. (2015). The deuterated variant $\mathrm{H}_{2}+\mathrm{OD} \rightarrow \mathrm{HDO}+\mathrm{H}$ can form $\mathrm{HDO}$ efficiently, but $\mathrm{D}_{2} \mathrm{O}$ formation is inefficient owing to the lower tunneling probability for the heavier $\mathrm{HD}$ and $\mathrm{D}_{2}$ molecules. Gasphase formation of water proceeds through ion-molecule reactions, namely, $\mathrm{D}_{2} \mathrm{O}$ is formed in the reaction $\mathrm{HD}_{2} \mathrm{O}^{+}+\mathrm{e}^{-} \rightarrow \mathrm{D}_{2} \mathrm{O}$ $+\mathrm{H}$ (see, e.g., van Dishoeck et al. 2014). Overall, the reactions leading to the formation of water and deuterated isotopologs are similar to the reference model.

The chemical models were subsequently compared using the 1D radiative hydrodynamics (RHD) model utilized in Furuya et al. (2016). The physical model follows a tracer particle during the collapse phase of a $3.9 \mathrm{M}_{\odot}$ core that forms a solar mass star; the simulation is explained in detail in Masunaga \& Inutsuka (2000). The initial conditions prior to the protostellar collapse are a 3 Myr static phase with $n_{\mathrm{H}}=2 \times 10^{4} \mathrm{~cm}^{-3}, T=10 \mathrm{~K}$, and an visual extinction of $A_{\mathrm{v}}=5 \mathrm{mag}$. The model is started from the atomic abundances detailed in Table 2. We adopted the ISRF of Draine (1978), corresponding to $F_{\mathrm{UV}} \sim 2 \times 10^{8}$ photons $\mathrm{cm}^{-2} \mathrm{~s}^{-1}$. The cosmic-ray induced UV flux is set to $3 \times 10^{3}$ photons $\mathrm{cm}^{-2} \mathrm{~s}^{-1}$. We define these initial conditions as our fiducial model; we return to the impact of the conditions during the static phase in subsequent sections. Figure 6 shows a comparison between the results obtained at the end of the simulation for a particle trajectory that reaches the protostar $(4.9 \mathrm{au})$ after $\sim 350 \mathrm{kyr}$. The final gas-phase ratios are comparable, with $f_{\mathrm{D} 1}$ of $1.3 \times 10^{-3}$ in the reference model, compared to $1.6 \times 10^{-3}$ the model used here. For $f_{\mathrm{D} 2}$, the results are $10^{-2}$ and $9.5 \times 10^{-3}$ for the reference model and this model, respectively. The minor difference in ratios are attributed to differences in the gas-phase network, where there is less formation of $\mathrm{HDO}$ and $\mathrm{D}_{2} \mathrm{O}$ compared with the reference model during the final stages of the collapse. The model presented here agrees with the chemical evolution of water proposed by Furuya et al. (2016), with lower D/H ratios in the mantle and higher $\mathrm{D} / \mathrm{H}$ ratios in the surface ice (see Figs. 6 and 8).
Table 2. Initial abundances following Aikawa et al. (2001), except for the inclusion of ortho and para spin-states of $\mathrm{H}_{2}$. The initial ortho/para ratio is motivated by the results of Furuya et al. (2016), who found that a ratio of 0.1 best reproduced the observed $\mathrm{D} / \mathrm{H}$ ratios in hot corinos toward low-mass protostars in their models.

\begin{tabular}{ll|ll}
\hline \hline Species & Abundance $\left(/ n_{\mathrm{H}}\right)$ & Species & Abundance $\left(/ n_{\mathrm{H}}\right)$ \\
\hline $\mathrm{o}-\mathrm{H}_{2}$ & $4.5(-1)$ & $\mathrm{S}+$ & $9.14(-8)$ \\
$\mathrm{p}-\mathrm{H}_{2}$ & $4.5(-2)$ & $\mathrm{Mg}+$ & $1.09(-8)$ \\
$\mathrm{He}$ & $9.75(-2)$ & $\mathrm{Si}+$ & $9.74(-9)$ \\
$\mathrm{HD}$ & $1.5(-5)$ & $\mathrm{Fe}+$ & $2.74(-9)$ \\
$\mathrm{O}$ & $1.8(-4)$ & $\mathrm{Na}+$ & $2.25(-9)$ \\
$\mathrm{C}+$ & $7.86(-5)$ & $\mathrm{P}+$ & $1(-9)$ \\
$\mathrm{H}$ & $5(-5)$ & $\mathrm{Cl}+$ & $2.16(-10)$ \\
$\mathrm{N}$ & $2.47(-5)$ & & \\
$A(B)=A \times 10^{B}$.
\end{tabular}

\section{Results}

\subsection{Comparing $1 D$ and $3 D$ models: $D / H$ ratios of water}

To establish how the chemical evolution of water differs between $1 \mathrm{D}$ and 3D models of the protostellar collapse, we compare the results of the 1D RHD model presented in Masunaga \& Inutsuka (2000) with the 3D MHD model presented in this work. These models share the same static phase of $3 \mathrm{Myr}$ as the fiducial model presented in the previous section. In Fig. 7, the evolution of gas-phase $f_{\mathrm{D} 1}$ and $f_{\mathrm{D} 2}$ during protostellar collapse are shown for a solar-mass protostar. The left panel shows the evolution for the 1D RHD model. The tracer particle reaches the hot corino $(T \gtrsim 100 \mathrm{~K})$ at $\tau \sim 10^{2}$, where $\tau=t_{\text {final }}-t$. Once in the hot corino, the complete desorption of the water ice lowers the $\mathrm{D} / \mathrm{H}$ ratios to $f_{\mathrm{D} 1} \sim 10^{-3}$ and $f_{\mathrm{D} 2} \sim 10^{-2}$, respectively, as the surface and mantle ice $\mathrm{D} / \mathrm{H}$ ratios are released and mixed with the gas-phase ratios. The right panel shows ten tracer particle trajectories from the RAMSES models accreted onto sink for the sink M101-C with a final mass of $1 \mathrm{M}_{\odot}$. In the figure, the shaded regions show the spread among the individual trajectories. In the 3D model, the gas-phase ratios show significant scatter during protostellar collapse and, generally, $f_{\mathrm{D} 1}$ and $f_{\mathrm{D} 2}$ are higher than in the 1D RHD model. The higher $\mathrm{D} / \mathrm{H}$ ratios are due to higher densities, which leads to more efficient gas-phase water deuteration. Once the particles reach the hot corino $\left(\tau \sim 10^{3}-10^{2}\right)$, the complete desorption of the water-ice reservoir reduces the variation drastically and the final ratios are similar for the ten tracer particles. The final $\mathrm{D} / \mathrm{H}$ ratio in the gas phase is primarily set by the $\mathrm{D} / \mathrm{H}$ ratio in the ice, which is similar among trajectories. To illustrate this, Fig. 8 shows the change in the ice composition for the same ten particle trajectories during the pre-collapse phase (100 kyr prior to formation of the protostar) and through the collapse. The selected tracer particles all reach the hot corino at $\sim 200-250 \mathrm{kyr}$ after the onset of the collapse. Initially, at $\tau \gtrsim 2.5 \times 10^{5} \mathrm{kyr}$, the $\mathrm{D} / \mathrm{H}$ ratios in both surface and mantle ice show significant scatter. This scatter is caused by variation in the densities in the early evolution of the trajectories. Once the trajectories reach the protostellar core, however, the $\mathrm{D} / \mathrm{H}$ ratios in the ice converge to similar values.

This change is driven by the convergence toward higher densities for the trajectories once they reach the dense core. This behavior is illustrated in Fig. 9, where the physical evolution of the ten particle trajectories is shown. The densities for the trajectories mainly range between $10^{5}-10^{6} \mathrm{~cm}^{-3}$ in the first $100 \mathrm{kyr}$, that is, around an order of magnitude higher than in the prestellar phase. At higher densities the thickness of the ice is increased. In 


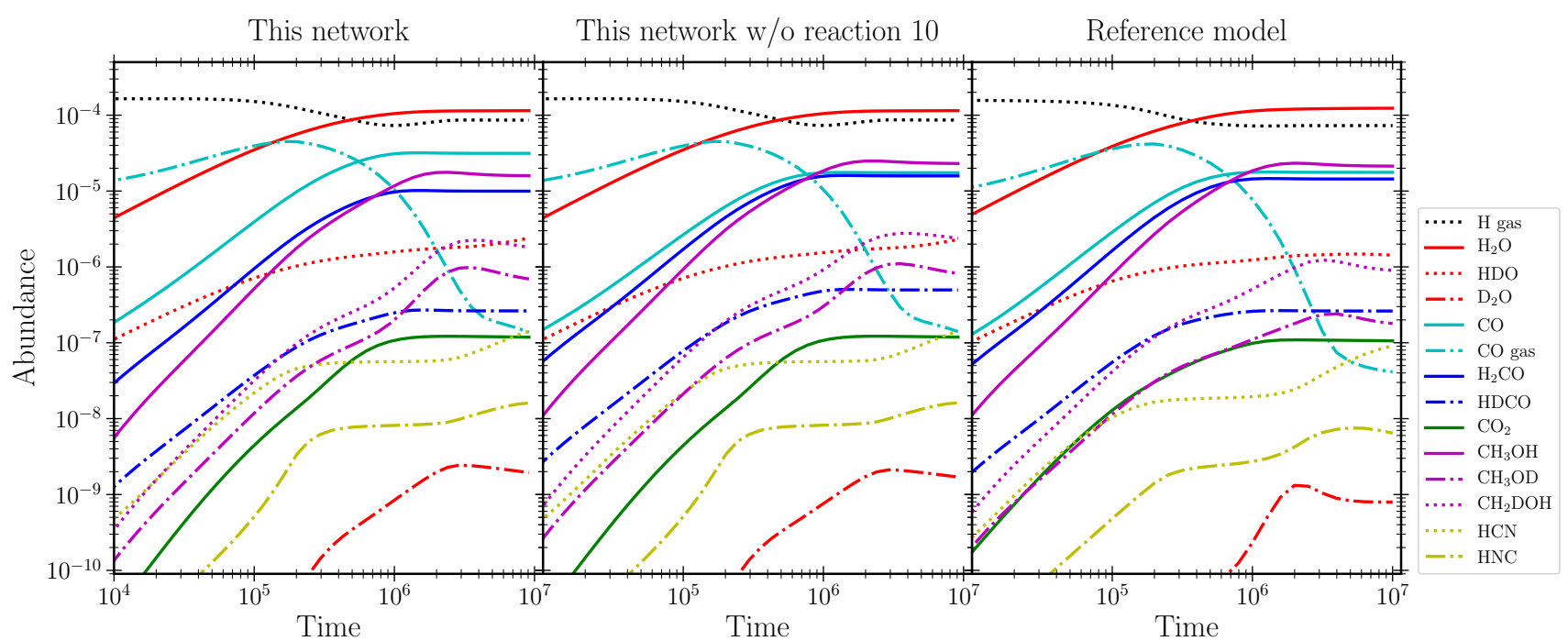

Fig. 5. Steady-state surface chemistry at $10 \mathrm{~K}, \mathrm{n}_{\mathrm{H}}=2 \times 10^{4}$, and $A_{\mathrm{v}}=5 \mathrm{mag}$. All species are bulk (mantle + surface) ice, expect when specified otherwise. The left panel shows the model used here with the inclusion of $\mathrm{CO}$ formation through reaction 10 while the middle panels show the network with this reaction switched off. The right panel shows the reference model from (Furuya et al. 2015.|2016)
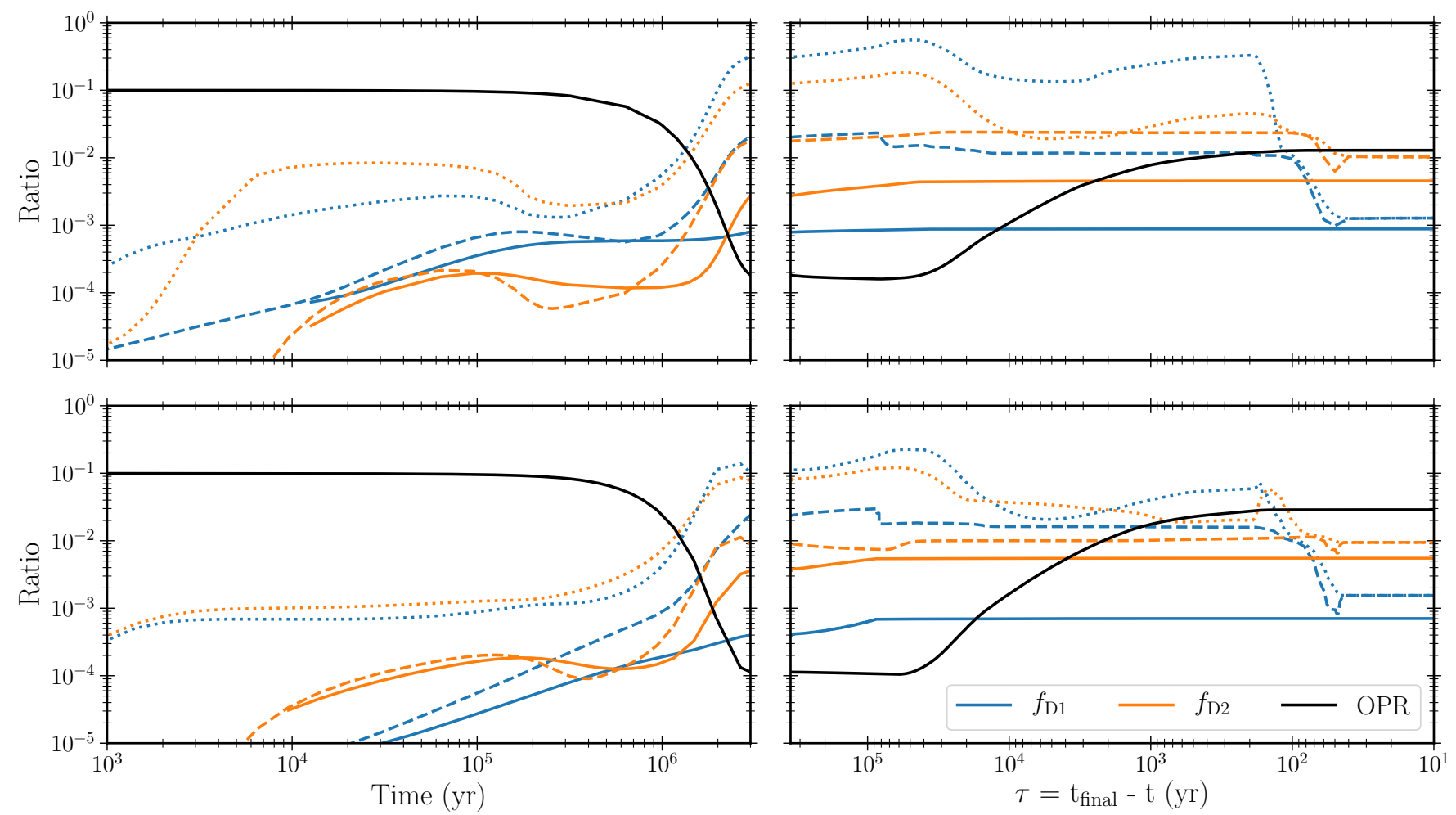

Fig. 6. Comparison between the chemical models presented in Furuya et al. (2016) (top panels) and the model utilized here (bottom panels). The 1D RHD simulations of a protostellar collapse are from Masunaga \& Inutsuka (2000). The solid lines indicate the ice mantle, the dashed lines present the ice surface, and the dotted lines show the gas-phase ratios in the three-phase models. The log-scale on the $\mathrm{x}$-axis is reversed on the second row.

the fiducial model this occurs at an epoch when water deuterium fractionation is highly efficient, which increases the $\mathrm{D} / \mathrm{H}$ ratio in the water ice. Generally, the densities within the protostellar cores are similar and this behavior is seen for all cores in the simulation, independent of mass and environment (see appendix C for the density profiles of the cores).
Toward the end of the collapse, the surface ice $f_{\mathrm{D} 1}$ varies up to a factor of $\sim 4$ between tracer trajectories, while $f_{\mathrm{D} 2}$ varies by less than a factor of 2. Meanwhile, in the mantle reservoirs, both $f_{\mathrm{D} 1}$ and $f_{\mathrm{D} 2}$ are almost constant between the tracer particle trajectories. We note that while the initial variations among trajectories are lost in Fig. 8, variations in the physical conditions 
in the static phase, still impact the final $\mathrm{D} / \mathrm{H}$ ratio as shown in the subsequent sections.

Overall, since the bulk ice show little variation among tracer particle trajectories in the fiducial model, a tracer particle accreted during the main accretion phase (e.g., during the Class 0 stage) shows little variation in the final $\mathrm{D} / \mathrm{H}$ ratio, if we assume the same initial conditions for all tracer particles.

\subsection{Comparing isolated and clusters protostars}

In Figs. 10 and 11 , we present comparisons between $f_{\mathrm{D} 1}$ for protostars with a similar final mass. The protostars labeled M048-I and M072-I are isolated, meaning that no neighboring protostars enter within 20,000 au of the protostar for the duration of the simulation. The remaining protostars are born in clusters; there are at least two neighboring protostars within 10,000 au of the protostars at some point during the simulation. Further characteristics of the protostars are presented in Table 1 .

The top panels show $f_{\mathrm{D} 1}$ in the gas phase as the tracer particles reach the hot corino region, that is, the gas-phase $\mathrm{HDO} / \mathrm{H}_{2} \mathrm{O}$ ratio once the water ice has been sublimated off of the dust grains. We focus on this region to provide ratios directly comparable to the ratios observed toward hot corinos (Jensen et al. 2019). Tracer particles are binned according to the time at which they reach the hot corino. A minor temporal evolution is evident; the tracer particles that accrete earlier showing slightly lower $\mathrm{D} / \mathrm{H}$ ratios than the tracer particles accreted toward the end of the simulation, but not to a degree where the effect is observationally resolvable. As mentioned in the previous section, the bulk of the ice reservoir remains similar for all tracer particles, limiting the degree to which the $\mathrm{D} / \mathrm{H}$ ratios can vary. The bottom panels of the figures show the $f_{\mathrm{D} 2}$, with an identical binning on the $\mathrm{x}$-axis. $f_{\mathrm{D} 2}$ shows less trend with time. This is attributed to the contemporary formation of the deuterated water isotopologs. As a longer accretion time leads to a higher overall $\mathrm{D} / \mathrm{H}$ ratio, the ratio of deuterated to non-deuterated water is increased, while the ratio between the two deuterated isotopologs remain roughly constant.

No significant differences in the $\mathrm{D} / \mathrm{H}$ ratios for the isolated and clustered protostars are seen in the simulations, in contrast with the observations presented in Jensen et al. (2019).

\subsection{Varying the physical conditions in the prestellar phase}

The results presented in Sect. 3.2 assume the same initial conditions at the onset of protostellar collapse. This assumption is unlikely to be fulfilled in dynamic star-forming regions, where temperature and radiation fields vary significantly depending on, for example, nearby massive stars and turbulence in the parental cloud. To assess the impact of the initial conditions, several combinations of initial conditions during the static phase were tested and are reported in Table 3

\subsubsection{Duration of prestellar phase}

In Fig. 12, we show the $f_{\mathrm{D} 1}$ and $f_{\mathrm{D} 2}$ for static phases with durations of $1 \mathrm{Myr}, 2 \mathrm{Myr}$, and $3 \mathrm{Myr}$. A shorter initial phase leads to a lower degree of deuterium fractionation. For a duration of $1 \mathrm{Myr}, f_{\mathrm{D} 1} \approx 4 \times 10^{-4}$ and $f_{\mathrm{D} 2} \approx 2 \times 10^{-3}$. Meanwhile, for a duration of $2 \mathrm{Myr}$, the $\mathrm{D} / \mathrm{H}$ ratios are increased and the ratios are closer to the $3 \mathrm{Myr}$ fiducial case, with $f_{\mathrm{D} 1} \approx 1 \times 10^{-3}$ and $f_{\mathrm{D} 2} \approx 2 \times 10^{-2}$. The shorter static phase of $1 \mathrm{Myr}$ introduces two additional effects: an increased scatter among different par- ticle trajectories, as indicated by the error bars in the figure, and a larger temporal variation among the tracer particles accreted early and late in the protostellar collapse. The latter effect is attributed to the shorter time to build the water ice, which has not reached a steady state at $1 \mathrm{Myr}$, making the chemical evolution more susceptible to variations in, for example, density, among the individual trajectories.

\subsubsection{Varying temperature}

Since the efficiency of deuterium fractionation depends on the temperature, several temperatures were tested in the prestellar phase. In Fig. 13, we show the results of increasing the temperature during the static phase. The radiation field and cosmicray flux are unaltered with respect to the fiducial model, and the prestellar duration is kept at $3 \mathrm{Myr}$. Increasing the temperature in the initial phase has only limited impact on the water $\mathrm{D} / \mathrm{H}$ ratio in the hot corino for a prestellar phase. While a higher temperature in the prestellar phase reduces the $f_{\mathrm{D} 1}$ and $f_{\mathrm{D} 2}$ in the ice prior to collapse, the $100 \mathrm{kyr}$ pre-collapse phase where the temperature is fixed at $10 \mathrm{~K}$ followed by low temperatures during the early phases of the protostellar collapse are sufficient to increase the $f_{\mathrm{D} 1}$ and $f_{\mathrm{D} 2}$ to $\gtrsim 10^{-3}$. The efficient deuterium fractionation on these short timescales is a consequence of the high densities in the cores $\left(n_{\mathrm{H}} \gtrsim 10^{5} \mathrm{~cm}^{-3}\right)$. For models \#13 and \#14 listed in Table 3 minimum temperatures of $15 \mathrm{~K}$ and $20 \mathrm{~K}$, respectively, were enforced during the protostellar collapse. In these cases, the final $\mathrm{D} / \mathrm{H}$ ratios in the hot corino remain lower, because the deuterium fractionation processes are less efficient throughout the entire chemical evolution. A stronger impact of higher temperatures is expected at temperatures $>30 \mathrm{~K}$, or at lower densities, where $\mathrm{CO}$ freeze-out is less efficient.

\subsubsection{Varying ISRF and cosmis-ray ionization rate}

Increasing the cosmic-ray ionization rate leads to a higher $f_{\mathrm{D} 1}$, from $\sim 10^{-3}$ at $\xi_{\mathrm{H} 2}=10^{-17} \mathrm{~s}^{-1}$, to $f_{\mathrm{D} 1}>10^{-2}$ at $\xi_{\mathrm{H} 2}=10^{-15} \mathrm{~s}^{-1}$. The impact on $f_{\mathrm{D} 2}$ is more complex. At $\xi_{\mathrm{H} 2}=10^{-16} \mathrm{~s}^{-1}, f_{\mathrm{D} 2}$ is slightly lower than the fiducial case, while at $\xi_{\mathrm{H} 2}=10^{-15} \mathrm{~s}^{-1}$, $f_{\mathrm{D} 2}$ is higher than the fiducial model early in the collapse and similar toward the end. Higher cosmic-ray ionization rates lead to a larger spread in the $\mathrm{D}_{2} \mathrm{O} / \mathrm{HDO}$ ratios between particles trajectories, as seen in Fig. 14 . The increased deuterium fractionation at stronger cosmic-ray fluxes is driven by the formation of $\mathrm{H}_{3}^{+}$and deuterated isotopologs, which are central to the fractionation processes in the cold ISM. The formation rates of $\mathrm{H}_{3}^{+}$ and isotopologs are regulated by the cosmic-ray ionization of $\mathrm{H}_{2}$ and consequently an increased cosmic-ray flux can increase rate of the fractionation process. Furthermore, higher ionization rates reduce the ortho-to-para conversion timescale for $\mathrm{H}_{2}$, which further enhances deuteration. The different formation pathways for $\mathrm{HDO}$ and $\mathrm{D}_{2} \mathrm{O}$ (i.e., HDO formation through surface reactions and $\mathrm{D}_{2} \mathrm{O}$ formation in the gas-phase) leads to the discrepancy between $f_{\mathrm{D} 1}$ and $f_{\mathrm{D} 2}$, as the efficiencies of the formation pathways differ.

Increasing the ISRF to $10 G_{0}$ in the static phase leads to a slight increase of $f_{\mathrm{D} 2}$, while $f_{\mathrm{D} 1}$ remains roughly constant. At $G=100 G_{0}, f_{\mathrm{D} 1}$ and $f_{\mathrm{D} 2}$ are increased compared with the fiducial model. These effects are due to different branching ratios for photodissociation of $\mathrm{HDO}$, where the branch producing $\mathrm{H}$ $+\mathrm{OD}$ is favored over the $\mathrm{D}+\mathrm{OH}$ branch. This leads to more efficient reformation of deuterated water, increasing deuterium fractionation through photodissociation in both gas-phase and 

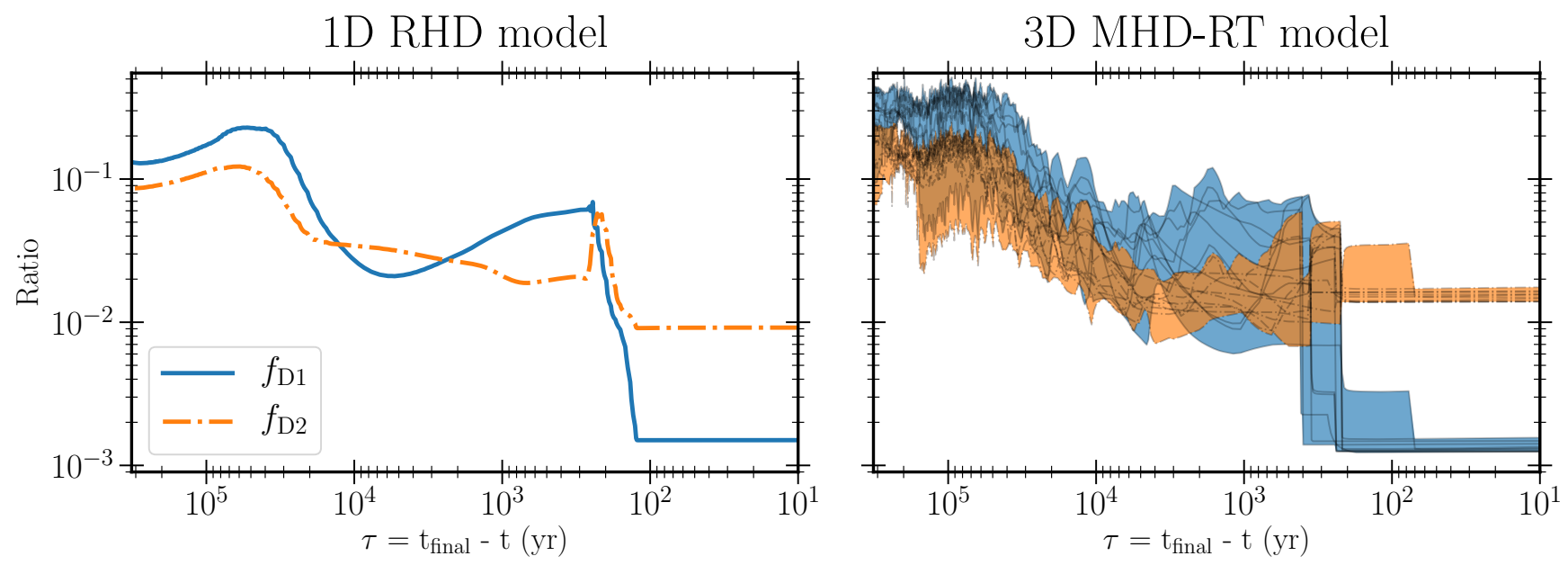

Fig. 7. Gas-phase evolution of $f_{\mathrm{D} 1}$ and $f_{\mathrm{D} 2}$ during protostellar collapse. The left panels shows the evolution of a particle accreted $\sim 350 \mathrm{kyr}$ after core collapse toward a $1 \mathrm{M}_{\odot}$ protostar. The right panel shows ten particle trajectories toward the $1 \mathrm{M}_{\odot}$ protostars M101-C in the 3D simulations.

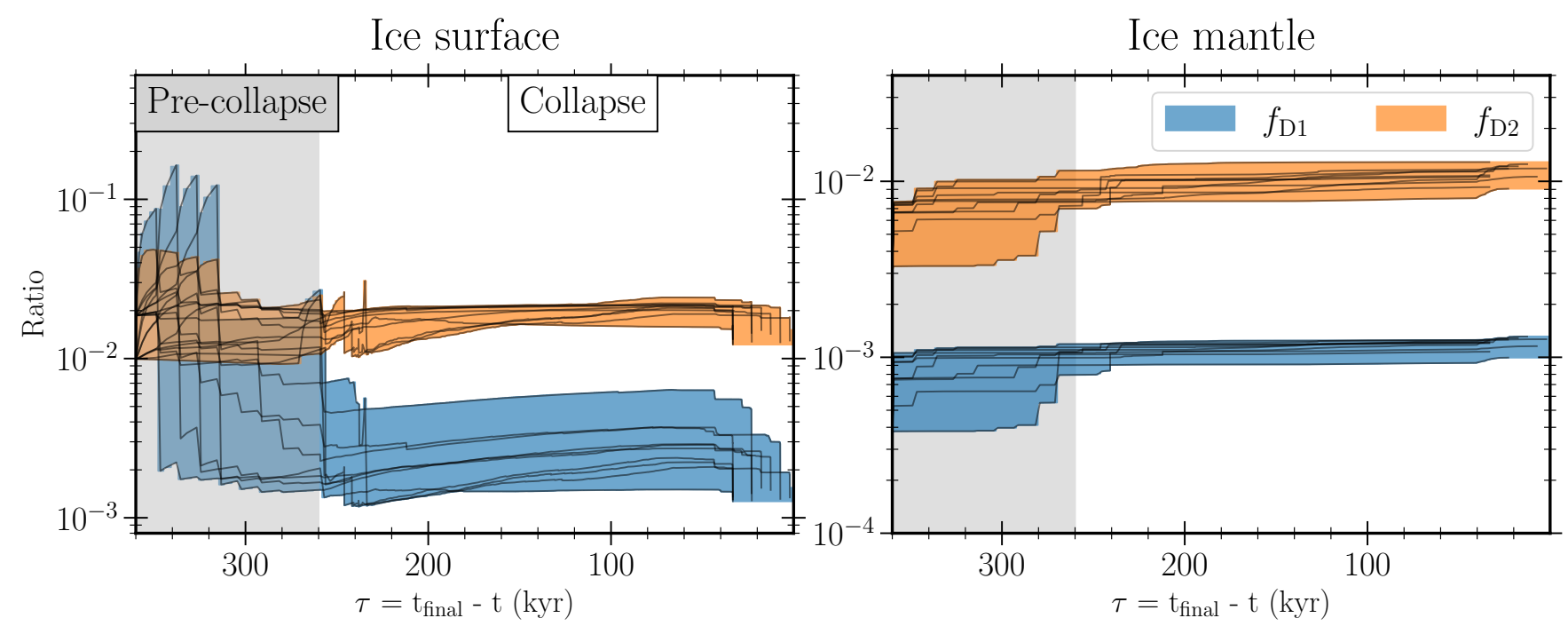

Fig. 8. Ice surface and mantle evolution for $f_{\mathrm{D} 1}$ and $f_{\mathrm{D} 2}$ during the protostellar collapse phase. The variation in densities for different trajectories in the pre-collapse phase induces large shifts in the ratios in the very beginning of the collapse. Once the tracer particles reach the protostellar core, the $\mathrm{D} / \mathrm{H}$ ratios in the ice mantle converge to similar values, as the trajectories reach similar densities. The y-axes differ between the left and right panels.

grain-surface chemistry (see, e.g., Arasa et al. 2015, and references therein). We note that a visual extinction of $A_{\mathrm{v}}=5 \mathrm{mag}$ was kept during the testing of initial conditions, and the ISRF hence remained heavily attenuated. At a lower visual extinction, stronger radiation fields lower the $\mathrm{D} / \mathrm{H}$ ratio, as $\mathrm{CO}$ freeze-out is reduced by photodesorption.

\subsubsection{Reproducing observed $\mathrm{HDO} / \mathrm{H}_{2} \mathrm{O}$ and $\mathrm{D}_{2} \mathrm{O} / \mathrm{HDO}$ ratios in hot corinos}

Current estimates of $f_{\mathrm{D} 1}$ and $f_{\mathrm{D} 2}$ in hot corinos of low-mass protostars are limited to a few sources. Coutens et al. (2014) detect $\mathrm{D}_{2} \mathrm{O}$ toward IRAS $2 \mathrm{~A}$ and derive $\alpha=f_{\mathrm{D} 2} / f_{\mathrm{D} 1} \gtrsim 7$. Similar high values of $\alpha$ are also seen for other protostars (S. Jensen et al., subm.). We require $\alpha \gtrsim 5$ and $f_{\mathrm{D} 1}$ in the range $10^{-4}-10^{-3}$ to reproduce current observations of the water $\mathrm{D} / \mathrm{H}$ ratios in hot corinos (Persson et al. 2014, Jensen et al. 2019). The fiducial model reproduced the observed $f_{\mathrm{D} 1} \sim 10^{-3}$ toward isolated protostars presented in Jensen et al. (2019), while the lower $\mathrm{D} / \mathrm{H}$ ratio toward the clustered protostars require different initial conditions. With the model presented in this work, reproducing $10^{-4}<f_{\mathrm{D} 1}<10^{-3}$ requires a shorter prestellar core phase or higher temperatures during the collapse. Model \#12, featuring a 1.5 Myr prestellar phase, yields $f_{\mathrm{D} 1} \sim 7 \times 10^{-4}$ and $\alpha \approx 13$, within the observed range for clustered sources. Similarly, models \#13 and \#14, where minimum temperatures of $15 \mathrm{~K}$ and $20 \mathrm{~K}$ are enforced during the entire collapse, yield $f_{\mathrm{D} 1} \sim 7 \times 10^{-4}$ and $f_{\mathrm{D} 1} \sim 3 \times 10^{-4}$, respectively, which is well within the observed ranges. These results are in line with the predictions from Jensen et al. (2019), who proposed that shorter timescale or higher temperatures could drive the observed differentiation between isolated and clustered protostars. Increasing the cosmic-ray flux changes $\alpha$ substantially, as shown in Table 3, and the observed $\mathrm{D} / \mathrm{H}$ ratios toward low-mass Class 0 protostars appear to be incompatible with higher cosmic-ray fluxes $\left(\xi_{\mathrm{H}_{2}}=10^{-15}\right)$. The 


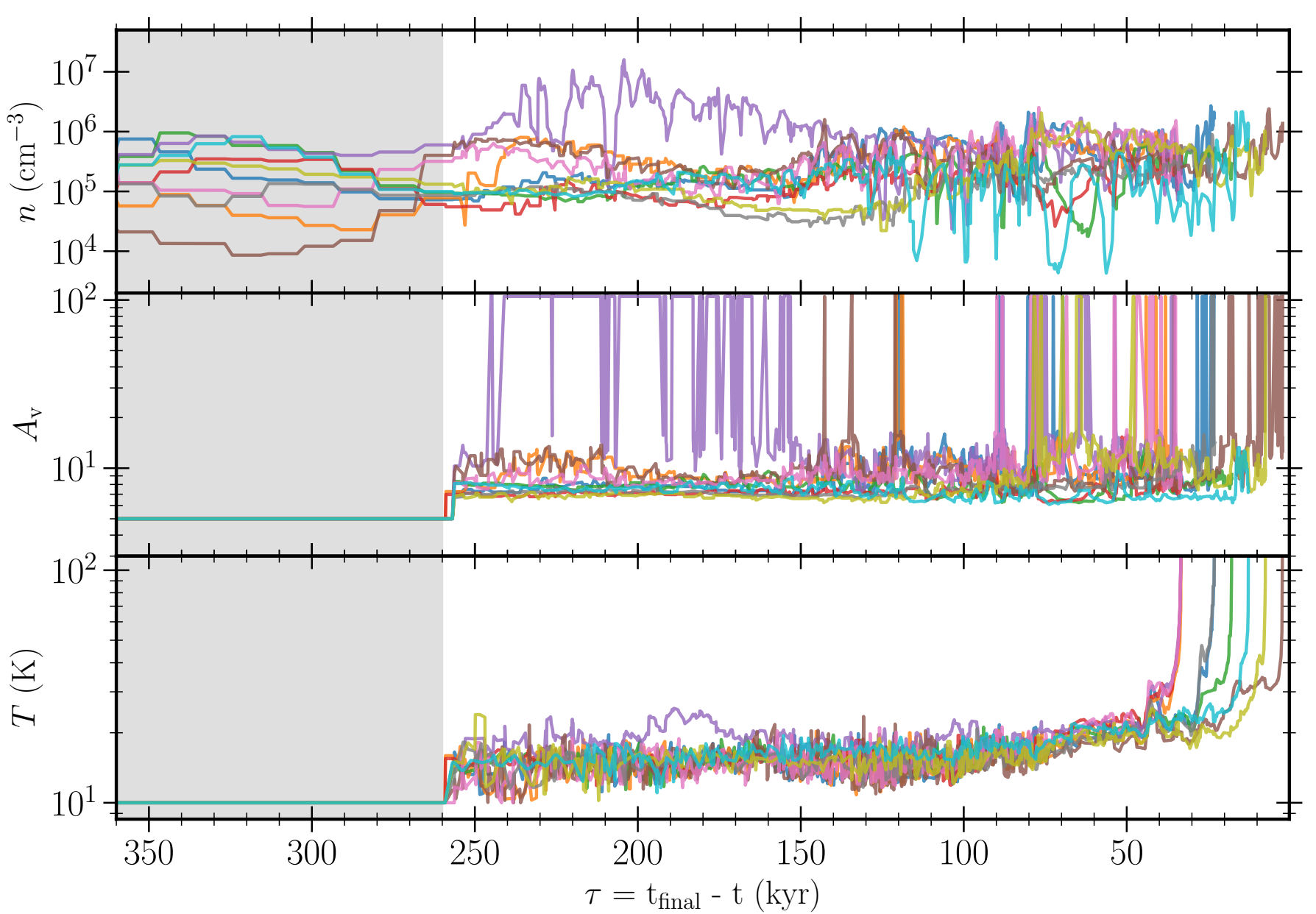

Fig. 9. Evolution in density, $A_{\mathrm{v}}$, and temperature for the ten tracer particles shown in Fig. 8 The shaded region indicates the 100 kyr pre-collapse phase, before the protostar is formed. In the pre-collapse phase, the densities are averaged in $10 \mathrm{kyr}$ windows, to reduce simulation time.

impact of a stronger ISRF on the $\mathrm{D} / \mathrm{H}$ ratios of water is limited within the model, where the visual extinction is relatively high during the entire simulation $\left(A_{\mathrm{v}} \gtrsim 5\right)$.

\section{Discussion}

\subsection{Impact of the environment during protostellar collapse}

The comparison of water $\mathrm{D} / \mathrm{H}$ ratios in realistic 3D protostellar collapse models show no significant variation between clustered and isolated protostars. The mass of the protostar does not appear to influence the water chemistry notably either. The homogenity of the $\mathrm{D} / \mathrm{H}$ ratios results from several factors. First, the physical structure of the low-mass protostellar cores presented in this work are similar; the density profiles resemble BonnorEbert spheres at the onset of collapse ${ }^{5}$ and once a tracer particle reaches the core, the $\mathrm{D} / \mathrm{H}$ ratios in the ice converge, independent of the surrounding environment. Second, the identical initial conditions in the model prior to the protostellar collapse

\section{C}

5 Radial density profiles for the protostars are presented in Appendix

Article number, page 12 of 23 limit the possible range of $\mathrm{D} / \mathrm{H}$ in the hot corino. Our fiducial model includes a $3 \mathrm{Myr}$ static core phase, during which the water $\mathrm{D} / \mathrm{H}$ ratio is established in the ice. Throughout the protostellar collapse, variations in tracer trajectories (e.g., $n(t)$ and $T(t))$ can induce variations in the gas-phase $\mathrm{D} / \mathrm{H}$ ratios, but the bulk water reservoir shows limited variation between trajectories and once the thick ice mantle is evaporated, the gas-phase $\mathrm{D} / \mathrm{H}$ ratio is largely similar to the ice $\mathrm{D} / \mathrm{H}$ ratio which is set by the physical conditions during ice formation (i.e., the evolution in the molecular cloud and static core).

While the observed differentiation in water deuteration toward isolated and clustered low-mass protostars is not explained by chemical processing during the protostellar collapse, the observed feature can be explained by variations in the physical evolution prior to protostellar collapse. A shorter static phase of $\lesssim 1.5$ Myr results in $f_{\mathrm{D} 1} \lesssim 7 \times 10^{-4}$, which agrees with the results toward clustered sources (Persson et al. 2014). Furthermore, these conditions produce $f_{\mathrm{D} 2} \sim 10^{-2}$, yielding $\alpha \gtrsim 5$ as observed toward IRAS 2A (Coutens et al. 2014). Increasing the temperature to $20 \mathrm{~K}$ during the entire collapse leads to a lower $f_{\mathrm{D} 1} \sim 3 \times 10^{-4}$, while $\alpha \sim 7$ is reproduced for a prephase dura- 


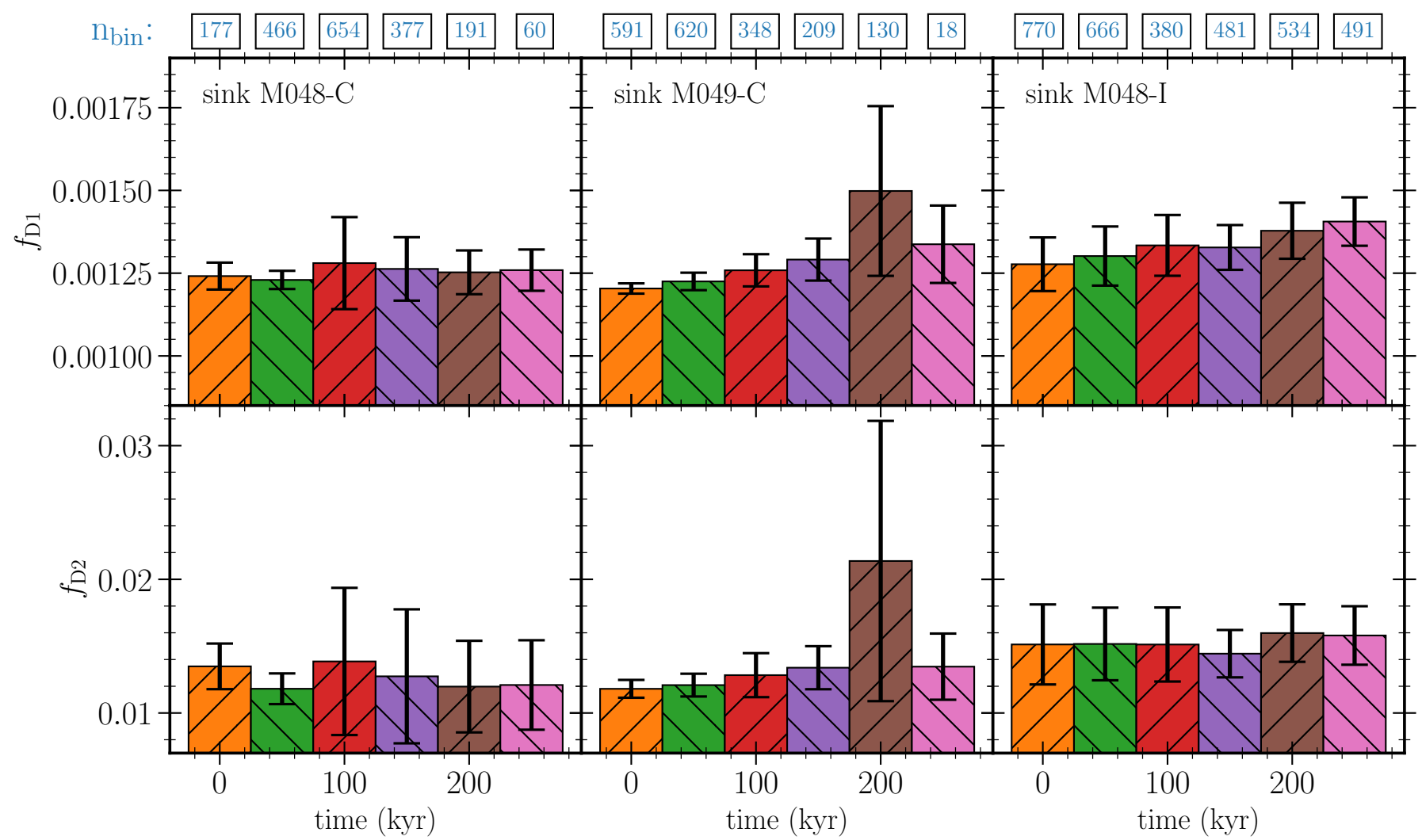

Fig. 10. $\mathrm{HDO} / \mathrm{H}_{2} \mathrm{O}\left(f_{\mathrm{D} 1}\right)$ and $\mathrm{D}_{2} \mathrm{O} / \mathrm{HDO}\left(f_{\mathrm{D} 2}\right)$ ratios toward three protostars in the simulation, with similar final mass of $\sim 0.5 \mathrm{M}_{\odot}$. The tracer particles are binned according to the time at which they reach the hot corino, in bins with a width of $50 \mathrm{kyr}$. The time corresponds to the time after the onset of collapse, $t_{0}$. The third protostar, sink M048-I, is classified as isolated since no protostar enters within 20,000 au during the simulation, while sink M048-C and sink M049-C are clustered. Each bar shows the median values of tracer particles accreted in the time interval, and the error shows the [15.9, 84.1] percentiles. The number of tracer particles within each bin is denoted in blue above the first row.

Table 3. Simulated $\mathrm{D} / \mathrm{H}$ ratios in the hot corino for a broad range of conditions in the prestellar stage. The reported $f_{\mathrm{D} 1}$ and $f_{\mathrm{D} 2}$ values are the median across all tracer particles entering the hot corino.

\begin{tabular}{llllllll}
\hline \hline$\#$ & $T(\mathrm{~K})$ & Duration $(\mathrm{Myr})$ & $\xi_{\mathrm{H} 2}\left(10^{-17} \mathrm{~s}^{-1}\right)$ & $G_{0}$ & $f_{\mathrm{D} 1}$ & $f_{\mathrm{D} 2}$ & $\alpha$ \\
\hline 1 & 10 & 1 & 1 & 1 & $5 \times 10^{-4}$ & $6 \times 10^{-3}$ & 12.0 \\
2 & 10 & 2 & 1 & 1 & $1 \times 10^{-3}$ & $2 \times 10^{-2}$ & 20.0 \\
3 & 10 & 3 & 1 & 1 & $1 \times 10^{-3}$ & $2 \times 10^{-2}$ & 20.0 \\
4 & 10 & 3 & 10 & 1 & $7 \times 10^{-3}$ & $1 \times 10^{-2}$ & 1.4 \\
5 & 10 & 3 & 100 & 1 & $3 \times 10^{-2}$ & $2 \times 10^{-2}$ & 0.7 \\
6 & 10 & 3 & 1 & 10 & $1 \times 10^{-3}$ & $2 \times 10^{-2}$ & 20.0 \\
7 & 10 & 3 & 1 & 100 & $3 \times 10^{-3}$ & $3 \times 10^{-2}$ & 10.0 \\
8 & 20 & 1 & 1 & 1 & $1 \times 10^{-3}$ & $1 \times 10^{-2}$ & 10 \\
9 & 30 & 1 & 1 & 1 & $2 \times 10^{-3}$ & $7 \times 10^{-3}$ & 3.5 \\
10 & 20 & 3 & 1 & 1 & $2 \times 10^{-3}$ & $3 \times 10^{-2}$ & 15.0 \\
11 & 30 & 3 & 1 & 1 & $1 \times 10^{-3}$ & $5 \times 10^{-3}$ & 5.0 \\
12 & 10 & 1.5 & 1 & 1 & $7 \times 10^{-4}$ & $8 \times 10^{-3}$ & 11.0 \\
$13^{a}$ & 15 & 1.5 & 1 & 1 & $7 \times 10^{-4}$ & $6 \times 10^{-3}$ & 8.6 \\
$14^{a}$ & 20 & 1.5 & 1 & 1 & $3 \times 10^{-4}$ & $2 \times 10^{-3}$ & 6.7 \\
\hline
\end{tabular}

Notes. ${ }^{a}$ For these models, the minimum temperature during the protostellar collapse was fixed at the temperature of the prestellar phase, as noted in the table.

tion of $>1.5$ Myr. Overall, temperatures in the range $T \lesssim 20 \mathrm{~K}$ can reproduce the observed $\mathrm{D} / \mathrm{H}$ ratios.

These models do not self-consistently predict the physical and chemical evolution during the molecular cloud and static core phase, however, both a shorter static phase and higher temperatures are feasible variations in dynamic molecular clouds. A shorter collapse timescale for clustered sources can be driven by higher densities or through external forces acceleration the collapse (e.g., Ward-Thompson et al. 2007; Enoch et al. 2008). Similarly, higher temperatures in clustered regions can occur through irradiation from nearby massive protostars or through shock heating in turbulent cloud environments (e.g., Krumholz 2014). 


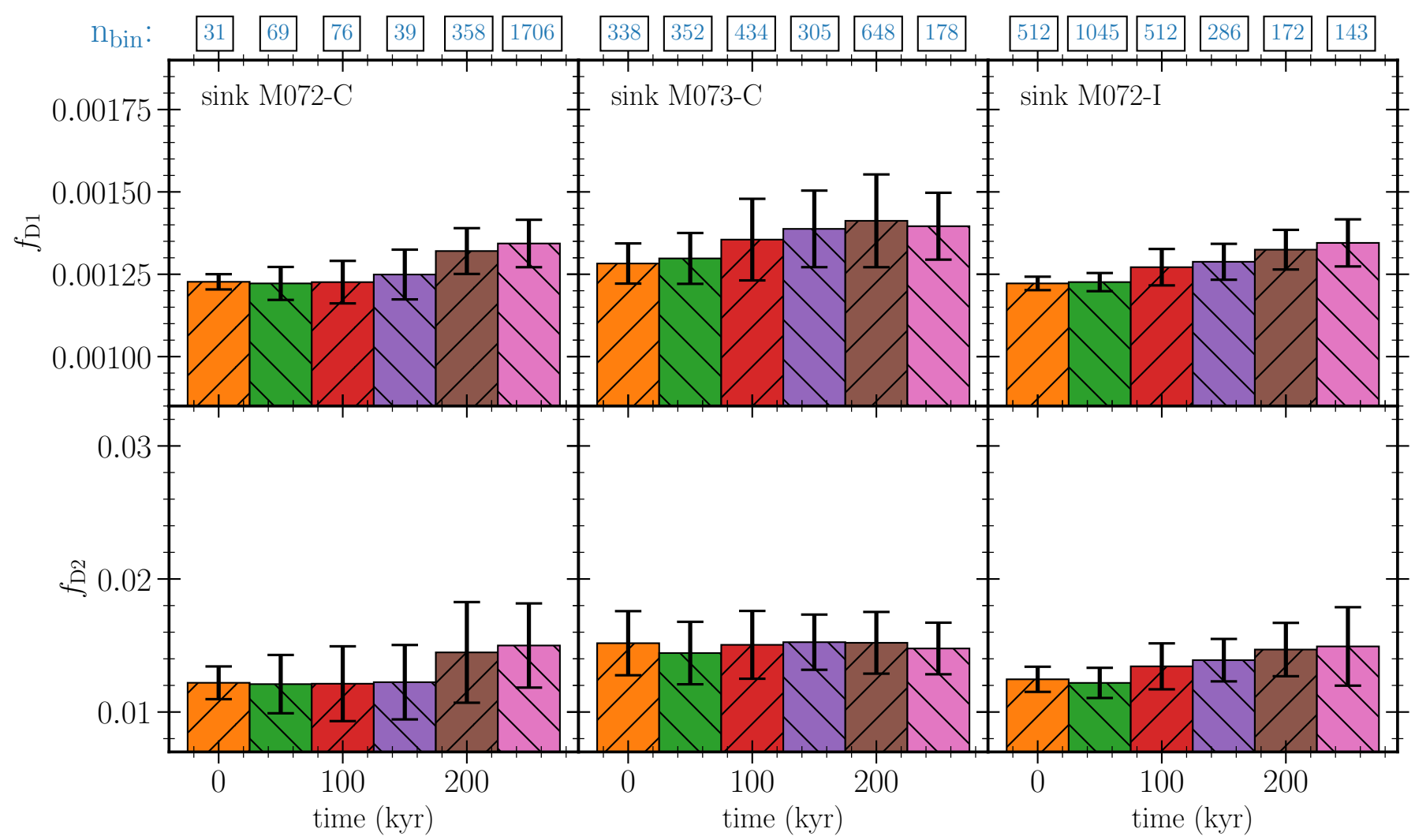

Fig. 11. Similar to Fig. 10 but for protostars with final masses of $\sim 0.72 \mathrm{M}_{\odot}$. The third protostar, sink M072-I, is classified as isolated since no protostar enters within 20,000 au during the simulation, while the remaining protostars are clustered.

\subsection{Caveats of the physical models}

Variations in cloud conditions prior to the protostellar collapse are not included in the models presented, except for a $100 \mathrm{kyr}$ period prior to the protostellar collapse where the temperature is fixed at $10 \mathrm{~K}$. Variations in the initial conditions are necessary to reproduce the observed variation in $\mathrm{D} / \mathrm{H}$ ratios among clustered and isolated protostars. To self-consistently model the conditions in the molecular cloud and static core phase, several enhancements to the model setup are required. Currently, radiative transfer is calculated locally around protostars once the protostellar collapse is initiated. The temperature in the gas prior to collapse is therefore unknown. Implementing global radiative transfer coupled with a thermochemical code, could provide better constraints on the temperature and radiation field during the molecular cloud and static phases (e.g., Glover et al. 2010, Grassi et al. 2014). To assess whether the fixed temperature of $10 \mathrm{~K}$ during the $100 \mathrm{kyr}$ pre-collapse phase influenced the results, we reran the fiducial models with variable temperatures and extinctions derived from parametric relations during this phase. The extinction was derived from the total number density through the relation:

$A_{\mathrm{v}}=\left(\frac{n_{\mathrm{H}}}{10^{3} \mathrm{~cm}^{-3}}\right)^{2 / 3} \operatorname{mag}$.

The above relation is derived in Grassi et al. (2017) by fitting a power law to the density-extinction relation in the RHD models of Glover et al. (2010). A semi-analytical relation between extinction and temperature is derived and tested in Hocuk et al. (2017) as follows:

$T_{\text {dust }}=11+5.7 \times \tanh \left[0.61-\log _{10}\left(A_{\mathrm{v}}\right)\right] \mathrm{K}$.
Implementing these relations during the pre-collapse phase had minimal effect on the final $\mathrm{D} / \mathrm{H}$ ratios and did not influence the conclusions presented in this work. This emphasizes that the $\mathrm{D} / \mathrm{H}$ ratio is predominantly a product of the initial conditions, with limited variation among different tracer particle trajectories.

The RAMSEs models are limited to by the grid resolution of 25 au. The resolution limit means that neither circumstellar disks nor outflows are sufficiently resolved. Resolving the physical structure of both outflow cavities and the disk is necessary to accurately predict the radiation and temperature field in the later stages of the protostellar formation. We are therefore limited to studying the initial collapse phase, where most of the matter is accreted. Increasing the resolution allows the disk and outflow cavities to be resolved and the results presented in Kuffmeier et al. (2018) demonstrate that disks are formed early on around the Class 0/I stage.

The three-phase model presented in this work does not consider bulk diffusion, that is, diffusion between surface and mantle or reactions within the ice mantle. Including bulk diffusion may impact the chemical evolution of three-phase models, especially during a protostellar collapse (Garrod 2013). Without bulk diffusion, molecules can remain locked in the ice mantle at temperatures exceeding their sublimation temperature, since only the surface molecules are free to sublimate. For instance, $\mathrm{CO}$ remains in the ice mantle at temperatures above $\sim 30 \mathrm{~K}$, since only the surface $\mathrm{CO}$ can desorb to the gas phase. This can alter the evolution of COMs and the change in CO gas-phase abundance; however, this does not impact the gas-phase deuterium fractionation process notable (Furuya et al. 2015). Furuya et al. (2017) study the evolution of $\mathrm{HDO} / \mathrm{H}_{2} \mathrm{O}$ and $\mathrm{D}_{2} \mathrm{O} / \mathrm{HDO}$ during protostellar collapse and find that the inclusion of bulk-diffusion does not impact the $\mathrm{D} / \mathrm{H}$ ratio of water post-collapse. Owing to 

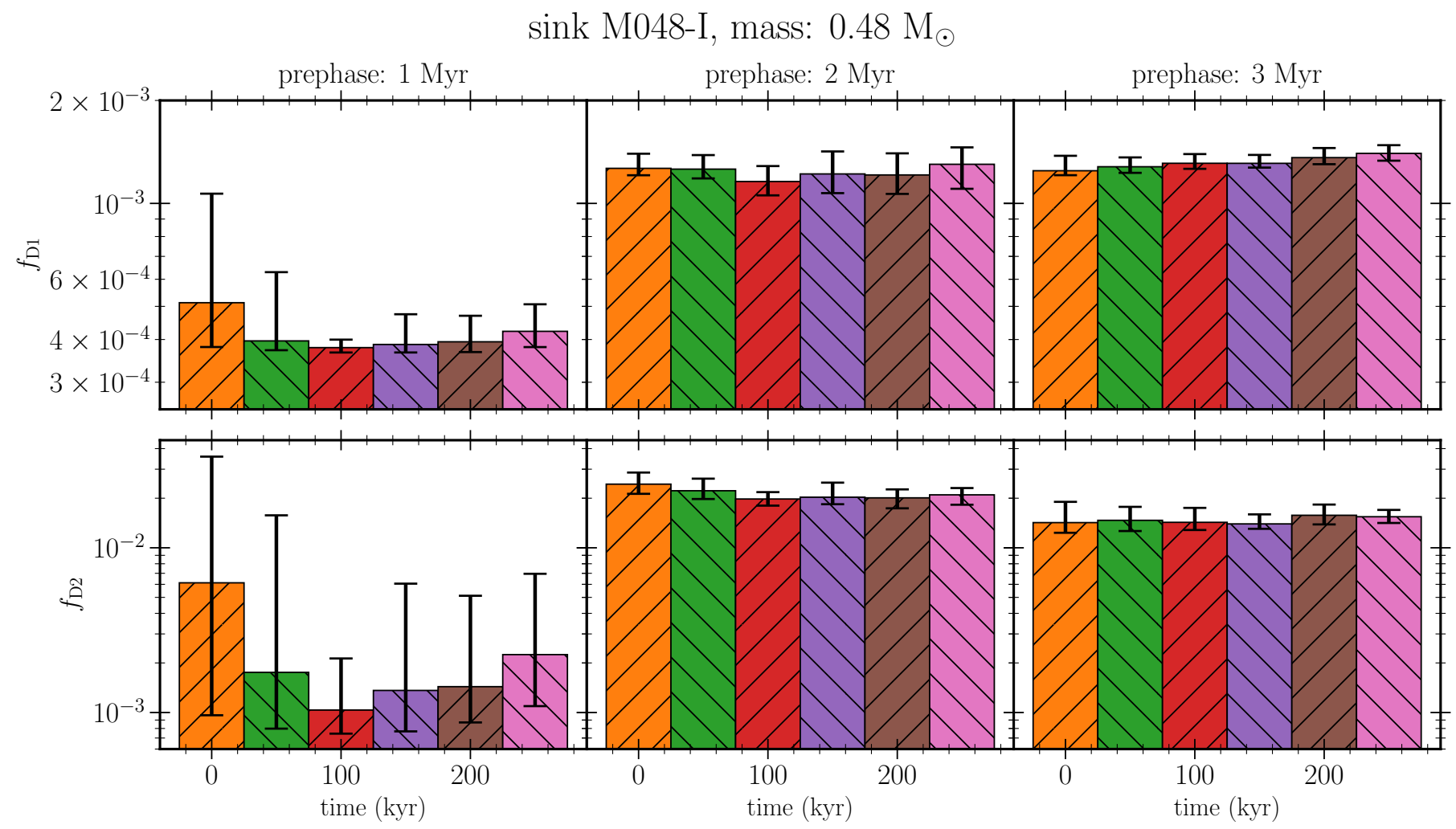

Fig. 12. $f_{\mathrm{D} 1}$ and $f_{\mathrm{D} 2}$ ratios toward the isolated protostar sink M048-I for varying prestellar phase durations. The tracer particles are binned according to the time at which they reach the hot corino, in bins with a width of $50 \mathrm{kyr}$. The time corresponds to the time after the onset of collapse, $t_{0}$. Each bar shows the median values of tracer particles accreted in the time interval, and the error shows the [15.9, 84.1] percentiles.

the high binding energy of water, bulk diffusion of water is likely inefficient and the effect is unlikely to alter the strong inheritance shown in the models.

\subsection{Inheritance of water $\mathrm{D} / \mathrm{H}$ ratio}

Determining whether the $\mathrm{D} / \mathrm{H}$ ratio is inherited or reset during star and planet formation is critical for our understanding of the evolution and delivery of water to young planets (e.g., van Dishoeck et al. 2014). Through simulations of water deuterium fractionation in a protoplanetary disk, Cleeves et al. (2014) showed that cometary $\mathrm{D} / \mathrm{H}$ ratios are too high to originate from local processing within the disk. Hence, some degree of inheritance is necessary to reproduce the deuterium fractionation in the Solar System, including the Earth's oceans. In a later work, Furuya et al. (2017) study the evolution of the D/H ratio of water, combining 1D protostellar collapse models with a 2D axisymmetric protoplanetary disk model. These authors find that the majority of the water in the protoplanetary disk remained unprocessed, again favoring inheritance for the majority of the water present in the disk. The results presented in this work favor chemical inheritance for the $\mathrm{D} / \mathrm{H}$ ratio of water in the hot corino as opposed to a "reset" chemistry post-collapse. Variation in physical evolution during the protostellar collapse has little impact on the degree of water deuteration in the hot corino, which is dominated by the conditions in the static phase. However, the $\mathrm{D} / \mathrm{H}$ ratio set in the static phase is not fixed and may evolve as the gas and dust enters the protostellar core, where densities increase. While this process adjusts the $\mathrm{D} / \mathrm{H}$ ratio in the ice, it is not a source of differentiation in the final $\mathrm{D} / \mathrm{H}$ ratios for water in the hot corinos since the structures of low-mass protostellar cores are largely similar between isolated and clustered protostars, owing to the physical processes that dominate the stability of cloud cores (e.g., Kuffmeier et al.2017).

Furuya et al. (2017) suggest that $\alpha$ is a better tool for distinguishing between inheritance and resetting of the deuterium fractionation, since their simulations yield $\alpha>1$ under prestellar conditions, while resetting of the chemistry in the protoplanetary disk leads to $\alpha<1$. Our results confirm that $\alpha>1$ in the case of inherited $\mathrm{D} / \mathrm{H}$ from the static phase when standard ISRF and cosmic-ray ionization rates are considered. This further motivates the study of $\mathrm{D}_{2} \mathrm{O} / \mathrm{HDO}$ ratios in hot corino to provide further constrains on the chemical evolution of water from cloud to core.

If the deuterium fractionation of water in the hot corino is inherited, the $\mathrm{D} / \mathrm{H}$ ratio of water at later stages in the star and planet formation process could be linked to the initial conditions in the cloud environment prior to the onset of star formation. However, the nature of hot corinos, and the chemical link from hot corino to protoplanetary disk is not well established at this stage (e.g., Belloche et al. 2020, Jørgensen et al. 2020). In a recent work, Coutens et al. (2020) study the chemical evolution from the collapse of a prestellar core to the formation of a protoplanetary disk. As in this work, the physical evolution during the collapse is tracked using tracer particles. Their model couples a 3D non-ideal MHD RAMSEs simulation of a collapsing core with the NAUTILUs chemical model and focuses on a broad range of molecules. These authors find a clear chemical link between the chemical composition in the prestellar core and in the protoplanetary disk. Generally, the principal elemental carriers remain the same throughout the collapse, with the exception of sulfur and phosphorus, where the main reser- 

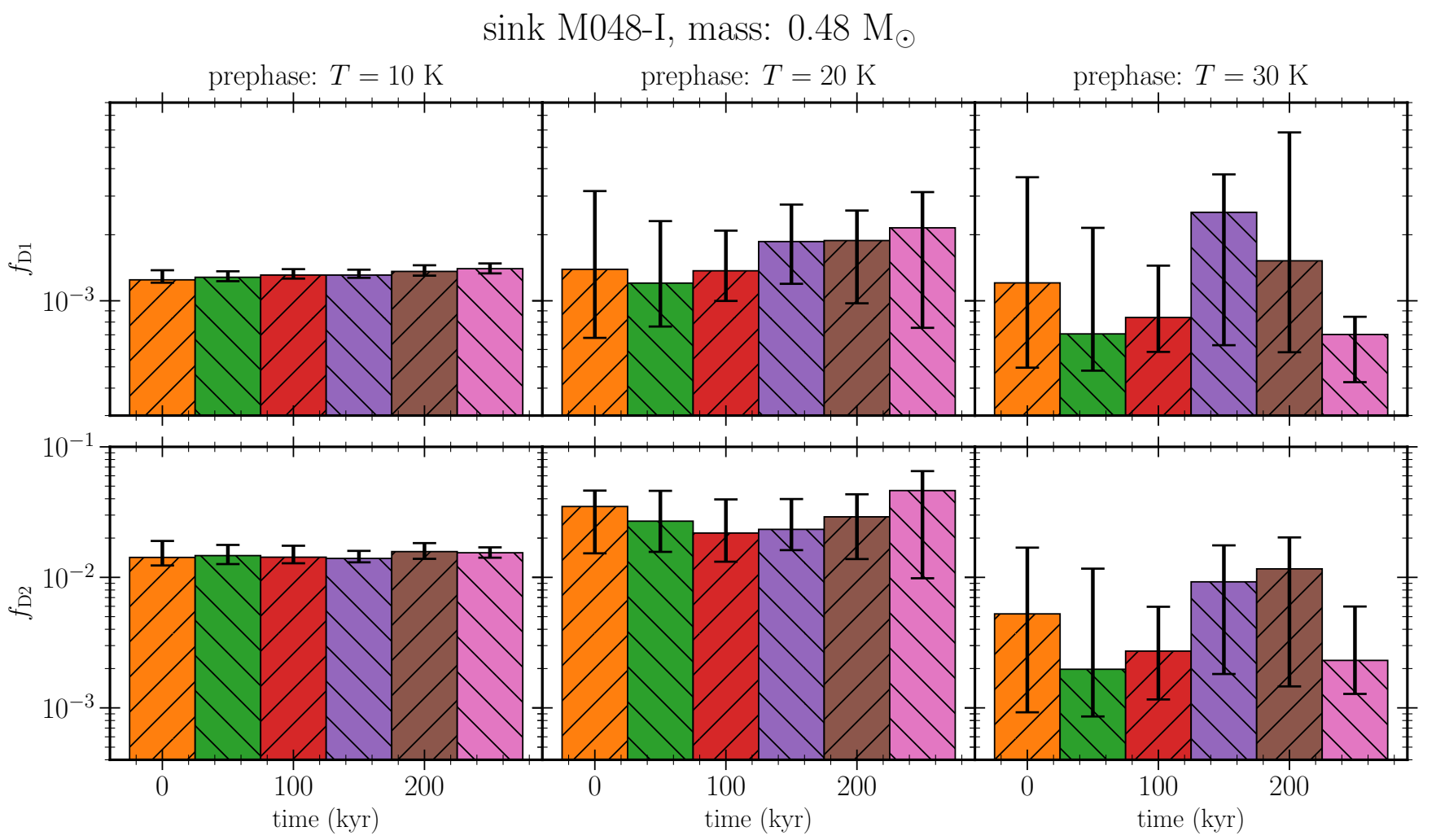

Fig. 13. $f_{\mathrm{D} 1}$ and $f_{\mathrm{D} 2}$ ratios toward the isolated protostar sink M048-I for varying temperatures during the prestellar phase. The tracer particles are binned according to the time at which they reach the hot corino, in bins with a width of $50 \mathrm{kyr}$. The time corresponds to the time after the onset of collapse, $t_{0}$. Each bar shows the median values of tracer particles accreted in the time interval, and the error shows the [15.9, 84.1] percentiles.

voirs evolve. Furthermore, the abundances of the most abundant molecules (e.g., $\mathrm{H}_{2} \mathrm{O}, \mathrm{CH}_{4}$, and $\mathrm{CH}_{3} \mathrm{OH}$ ) remain largely unaltered through the collapse, although the abundance of "large" COMs (e.g., $\mathrm{CH}_{3} \mathrm{CHO}, \mathrm{CH}_{3} \mathrm{OCH}_{3}$ ) increases during the warmup phase. Overall, these conclusions agree with the results presented in this work for water, namely, that the initial conditions have a high impact on the final chemical composition; this supports a high degree of inheritance during the protostellar collapse.

In recent years, rotational signatures have been observed in a limited sample of young embedded Class 0 sources (e.g., Tobin et al. 2012; Murillo et al. 2013), including one source hosting a hot corino (Bjerkeli et al. 2019, Imai et al. 2019). This could suggest that the chemistry observed in hot corinos reflects the same chemical reservoir that constitutes the initial disk structure. Still, detections of the warm gas component in sources with clear disk structures remains elusive (e.g., Artur de la Villarmois et al. 2018). Furthermore, once the disk is formed, in situ chemical processes may locally alter the chemical composition throughout the disk, introducing chemical gradients in the radial and vertical directions (e.g., Henning \& Semenov 2013). Recently, a correlation between COMs in the hot corino of IRAS162932422 and the comet 67P were established, supporting a potential link between the chemical composition of the hot corino and protoplanetary disk, where comets are formed (Drozdovskaya et al. 2019). This could imply limited chemical processing in the protosolar nebula at larger orbital distances where comets were formed. Furthermore, a recent study of COMs in the disk around V883 Ori found similarities with the chemistry in hot corinos and comets (Lee et al. 2019).

\section{Summary}

In this work we present physicochemical models of protostellar collapse to study how variations in protostellar cloud environments may impact the chemistry, specifically the $\mathrm{D} / \mathrm{H}$ ratio of water. The main results are as follows:

- The $\mathrm{HDO} / \mathrm{H}_{2} \mathrm{O}$ and $\mathrm{D}_{2} \mathrm{O} / \mathrm{HDO}$ ratios show no clear correlation with the cloud environment. Physical variations during the protostellar collapse are not sufficient to drive a notable difference in the deuterium fractionation between different protostars as the $\mathrm{D} / \mathrm{H}$ ratio is governed by the initial cloud conditions prior to protostellar collapse.

- To reproduce the observed differentiation in $\mathrm{HDO} / \mathrm{H}_{2} \mathrm{O}$ ratios between isolated and clustered protostar, variations in the duration or temperature of the prestellar phase are necessary. As such, the $\mathrm{D} / \mathrm{H}$ ratio is determined by these conditions, favoring inheritance of the water $\mathrm{D} / \mathrm{H}$ ratio observed in hot corinos. Static phases with durations of 1$3 \mathrm{Myr}$ and temperatures 10-20 K can reproduce the observed $\mathrm{HDO} / \mathrm{H}_{2} \mathrm{O}$ and $\mathrm{D}_{2} \mathrm{O} / \mathrm{HDO}$ ratios.

- Increasing the cosmic-ray ionization rates to $\xi_{\mathrm{H} 2} \approx 10^{-15} \mathrm{~s}^{-1}$ leads to $\mathrm{D} / \mathrm{H}$ ratios that are incompatible with the observed values in hot corinos toward low-mass Class 0 sources.

- Global chemical models of star-forming regions are needed to establish the origin of chemical differentiation in starforming regions. Tracing the chemical evolution from molecular cloud to hot corino is a necessary step to selfconsistently determine which processes drive chemical differentiation of water and more complex molecules.

This work illustrates how isotope fractionation can trace the chemical evolution through the star and planet formation process, by combining detailed models with the latest observational 

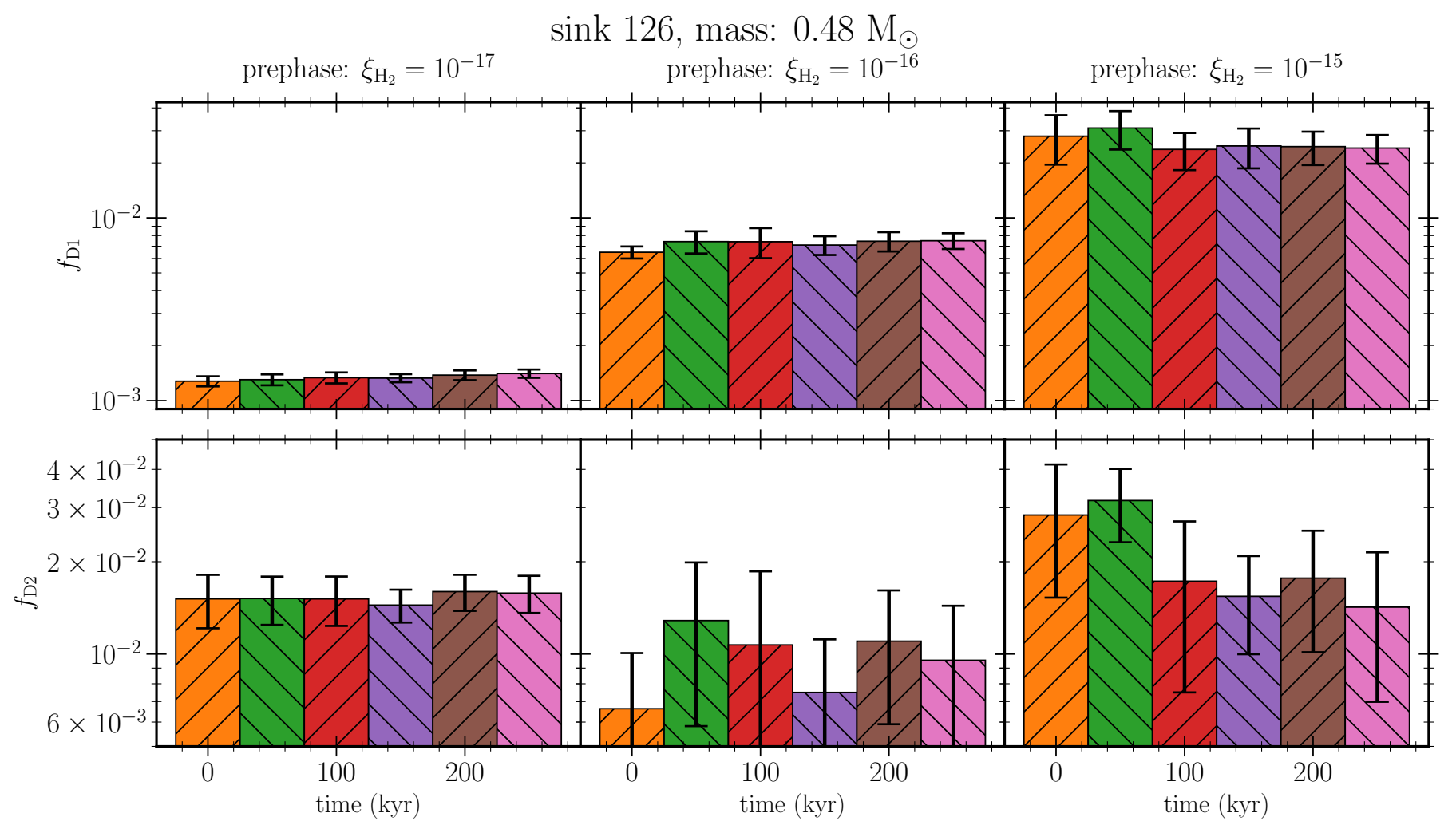

Fig. 14. $f_{\mathrm{D} 1}$ and $f_{\mathrm{D} 2}$ ratios toward the isolated protostar sink M048-I for cosmic-ray ionization rates $\xi_{\mathrm{H} 2}$. The tracer particles are binned according to the time at which they reach the hot corino, in bins with a width of $50 \mathrm{kyr}$. The time corresponds to the time after the onset of collapse, $t_{0}$.

advances. Furthermore, present results highlight the need for physicochemical models spanning the entire star formation process, to accurately model the origin and evolution of chemical complexity during the formation of stars and planets.

Acknowledgements. The authors are grateful to Tommaso Grassi, who provided the underlying framework for the chemical model. The authors would like to thank the referee for constructive comments which helped improve the manuscript. The group of JKJ acknowledges support from the European Research Council (ERC) under the European Union's Horizon 2020 research and innovation programme (grant agreement No 646908) through ERC Consolidator Grant "S4F". TH was supported by the Independent Research Fund Denmark through grant No. DFF 8021-00350B. We acknowledge PRACE for awarding us access to Curie at GENCI@CEA, France, and to Marconi at CINECA, Italy used to carry out the simulation. Resources at the University of Copenhagen HPC centre, funded in part by the Carlsberg, Novo, and Villum foundations, were used for the data analysis. This work made use of MATPLOTLIB (Hunter 2007) and NUMPY (Harris et al.2020).

\section{References}

Aikawa, Y., Furuya, K., Yamamoto, S., \& Sakai, N. 2020, ApJ, 897, 110 Aikawa, Y., Ohashi, N., Inutsuka, S.-i., Herbst, E., \& Takakuwa, S. 2001, ApJ 552,639

Aikawa, Y., Wakelam, V., Hersant, F., Garrod, R. T., \& Herbst, E. 2012, ApJ, 760, 40

Altwegg, K., Balsiger, H., Berthelier, J. J., et al. 2017, Philosophical Transactions of the Royal Society of London Series A, 375, 20160253

Arasa, C., Koning, J., Kroes, G.-J., Walsh, C., \& van Dishoeck, E. F. 2015, A\&A, 575, A 121

Artur de la Villarmois, E., Kristensen, L. E., Jørgensen, J. K., et al. 2018, A\&A, 614, A 26

Belloche, A., Maury, A. J., Maret, S., et al. 2020, A\&A, 635, A198

Bjerkeli, P., Ramsey, J. P., Harsono, D., et al. 2019, A\&A, 631, A64

Bohlin, R. C., Savage, B. D., \& Drake, J. F. 1978, ApJ, 224, 132

Caselli, P., Keto, E., Bergin, E. A., et al. 2012, ApJ, 759, L37

Caselli, P., Stantcheva, T., Shalabiea, O., Shematovich, V. I., \& Herbst, E. 2002, Planet. Space Sci., 50, 1257
Caselli, P., van der Tak, F. F. S., Ceccarelli, C., \& Bacmann, A. 2003, A\&A, 403, L37

Chuang, K. J., Fedoseev, G., Ioppolo, S., van Dishoeck, E. F., \& Linnartz, H. 2016, MNRAS, 455, 1702

Cleeves, L. I., Bergin, E. A., Alexand er, C. M. O. D., et al. 2014, Science, 345, 1590

Coutens, A., Commerçon, B., \& Wakelam, V. 2020, A\&A, 643, A108

Coutens, A., Jørgensen, J. K., Persson, M. V., et al. 2014, ApJ, 792, L5

Coutens, A., Vastel, C., Cabrit, S., et al. 2013, A\&A, 560, A39

Cuppen, H. M., Walsh, C., Lamberts, T., et al. 2017, Space Sci. Rev., 212, 1

D'Antona, F. \& Mazzitelli, I. 1997, Mem. Soc. Astron. Italiana, 68, 807

Diplas, A. \& Savage, B. D. 1994, ApJ, 427, 274

Draine, B. T. 1978 , ApJS, 36, 595

Draine, B. T. \& Bertoldi, F. 1996, ApJ, 468, 269

Drozdovskaya, M. N., van Dishoeck, E. F., Rubin, M., Jørgensen, J. K., \& Altwegg, K. 2019, MNRAS, 490, 50

Dullemond, C. P., Juhasz, A., Pohl, A., et al. 2012, RADMC-3D: A multipurpose radiative transfer tool

Enoch, M. L., Evans, Neal J., I., Sargent, A. I., et al. 2008, ApJ, 684, 1240

Fayolle, E. C., Bertin, M., Romanzin, C., et al. 2011, ApJ, 739, L36

Fayolle, E. C., Bertin, M., Romanzin, C., et al. 2013, A\&A, 556, A122

Frimann, S., Jørgensen, J. K., \& Haugbølle, T. 2016, A\&A, 587, A59

Furuya, K., Aikawa, Y., Hincelin, U., et al. 2015, A\&A, 584, A124

Furuya, K., Drozdovskaya, M. N., Visser, R., et al. 2017, A\&A, 599, A40

Furuya, K., van Dishoeck, E. F., \& Aikawa, Y. 2016, A\&A, 586, A127

Garrod, R. T. 2013, ApJ, 765, 60

Garrod, R. T. \& Herbst, E. 2006, A\&A, 457, 927

Garrod, R. T. \& Pauly, T. 2011, ApJ, 735, 15

Garrod, R. T., Wakelam, V., \& Herbst, E. 2007, A\&A, 467, 1103

Glover, S. C. O., Federrath, C., Mac Low, M. M., \& Klessen, R. S. 2010, MNRAS, 404, 2

Goldsmith, P. F. 2001, ApJ, 557, 736

Grassi, T., Bovino, S., Haugbølle, T., \& Schleicher, D. R. G. 2017, MNRAS, 466, 1259

Grassi, T., Bovino, S., Schleicher, D. R. G., et al. 2014, MNRAS, 439, 2386

Harris, C. R., Millman, K. J., van der Walt, S. J., et al. 2020, Nature, 585, 357-362

Hasegawa, T. I. \& Herbst, E. 1993, MNRAS, 261, 83

Hasegawa, T. I., Herbst, E., \& Leung, C. M. 1992, ApJS, 82, 167

Haugbølle, T., Padoan, P., \& Nordlund, A. 2018, ApJ, 854, 35

He, J., Acharyya, K., \& Vidali, G. 2016, ApJ, 823, 56 
sink M048-I, mass: $0.48 \mathrm{M}_{\odot}$
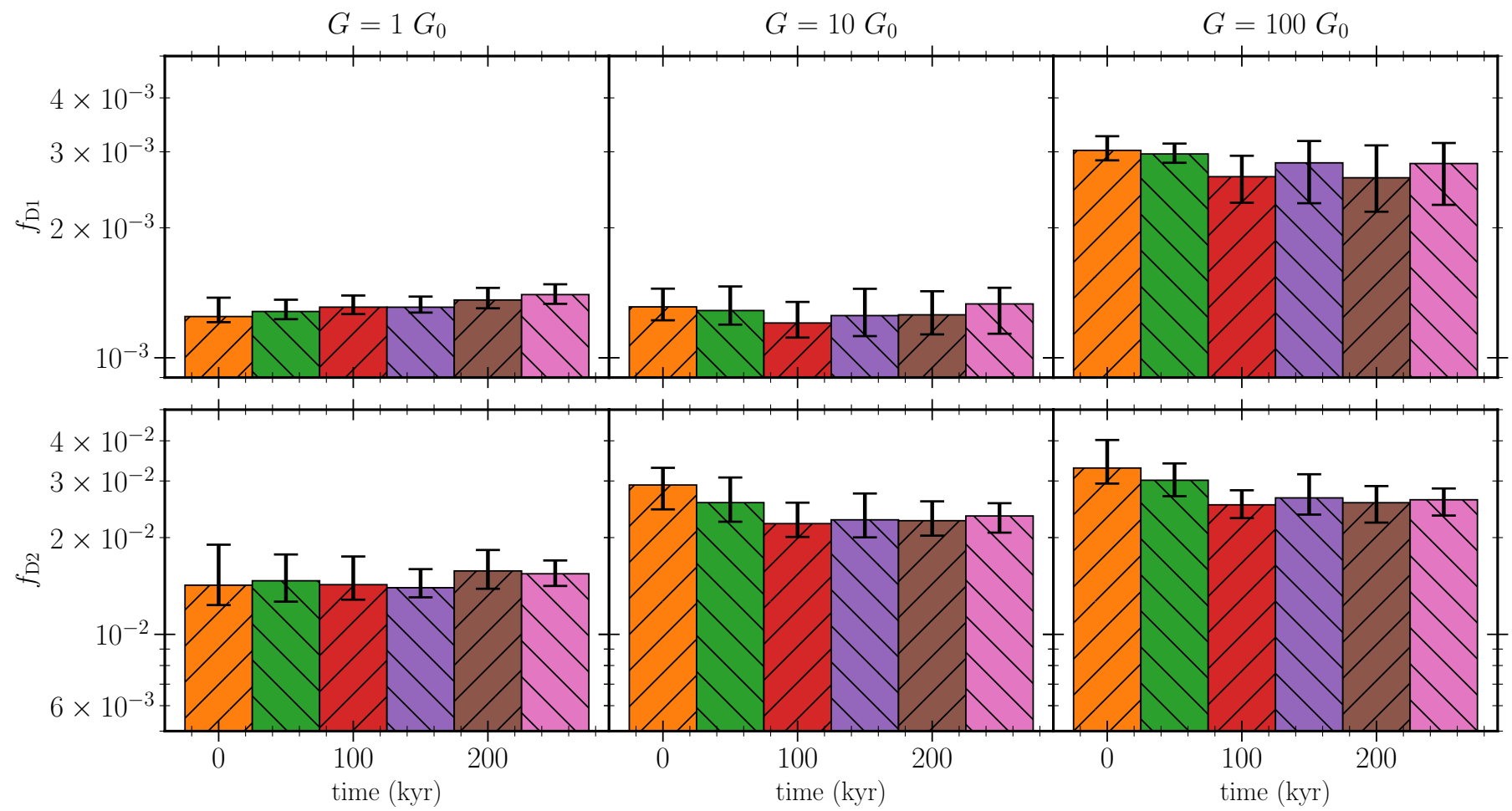

Fig. 15. $f_{\mathrm{D} 1}$ and $f_{\mathrm{D} 2}$ ratios toward the isolated protostar sink M048-I for varying ISRF, $1 G_{0}$ corresponds to the ISRF of Draine (1978). Tracer particles are binned according to the time at which they reach the hot corino, in bins with a width of 50 kyr. Time corresponds to the time after the onset of collapse, $t_{0}$. Each bar shows the median values of tracer particles accreted in the time interval, and the error shows the [15.9, 84.1] percentiles.

Heays, A. N., Bosman, A. D., \& van Dishoeck, E. F. 2017, A\&A, 602, A105 Heays, A. N., Visser, R., Gredel, R., et al. 2014, A\&A, 562, A61 Henning, T. \& Semenov, D. 2013, Chemical Reviews, 113, 9016 Hocuk, S., Szúcs, L., Caselli, P., et al. 2017, A\&A, 604, A58 Hugo, E., Asvany, O., \& Schlemmer, S. 2009, J. Chem. Phys., 130, 164302 Hunter, J. D. 2007, Computing in Science \& Engineering, 9, 90 Imai, M., Oya, Y., Sakai, N., et al. 2019, ApJ, 873, L21

Jensen, S. S. \& Haugbølle, T. 2018, MNRAS, 474, 1176

Jensen, S. S., Jørgensen, J. K., Kristensen, L. E., et al. 2019, A\&A, 631, A25 Jørgensen, J. K., Belloche, A., \& Garrod, R. T. 2020, Annual Review of Astronomy and Astrophysics, 58, null

Keto, E. \& Caselli, P. 2008, ApJ, 683, 238

Krumholz, M. R. 2014, Phys. Rep., 539, 49

Kuffmeier, M., Frimann, S., Jensen, S. S., \& Haugbølle, T. 2018, MNRAS, 475, 2642

Kuffmeier, M., Haugbølle, T., \& Nordlund, A. 2017, ApJ, 846, 7

Lee, J.-E., Lee, S., Baek, G., et al. 2019, Nature Astronomy, 3, 314

Leger, A., Jura, M., \& Omont, A. 1985, A\&A, 144, 147

Linsky, J. L. 2003, Space Sci. Rev., 106, 49

Majumdar, L., Gratier, P., Ruaud, M., et al. 2017, MNRAS, 466, 4470

Masunaga, H. \& Inutsuka, S.-i. 2000, ApJ, 531, 350

Murillo, N. M., Lai, S.-P., Bruderer, S., Harsono, D., \& van Dishoeck, E. F. 2013, A\&A, 560, A103

Oberg, K. I., Fuchs, G. W., \& van Dishoeck, E. F. 2007, in Molecules in Space and Laboratory, ed. J. L. Lemaire \& F. Combes, 80

Öberg, K. I., van Dishoeck, E. F., \& Linnartz, H. 2009, A\&A, 496, 281

Pagani, L., Bacmann, A., Cabrit, S., \& Vastel, C. 2007, A\&A, 467, 179

Pagani, L., Salez, M., \& Wannier, P. G. 1992, A\&A, 258, 479

Parise, B., Caux, E., Castets, A., et al. 2005, A\&A, 431, 547

Persson, M. V., Jørgensen, J. K., van Dishoeck, E. F., \& Harsono, D. 2014, A\&A, 563, A74

Richings, A. J., Schaye, J., \& Oppenheimer, B. D. 2014, MNRAS, 442, 2780

Rodgers, S. D. \& Charnley, S. B. 2002, Planet. Space Sci., 50, 1125

Ruaud, M., Wakelam, V., \& Hersant, F. 2016, MNRAS, 459, 3756

Semenov, D., Henning, T., Helling, C., Ilgner, M., \& Sedlmayr, E. 2003, A\&A, 410,611

Shingledecker, C. N., Vasyunin, A., Herbst, E., \& Caselli, P. 2019, ApJ, 876, 140

Sipilä, O., Caselli, P., \& Harju, J. 2015, A\&A, 578, A55
Taquet, V., Peters, P. S., Kahane, C., et al. 2013, A\&A, 550, A127

Teyssier, R. 2002, A\&A, 385, 337

Tielens, A. G. G. M. \& Allamandola, L. J. 1987, Composition, Structure, and Chemistry of Interstellar Dust, ed. D. J. Hollenbach \& J. Thronson, Harley A., Vol. 134, 397

Tobin, J. J., Hartmann, L., Chiang, H.-F., et al. 2012, Nature, 492, 83

van Dishoeck, E. F., Bergin, E. A., Lis, D. C., \& Lunine, J. I. 2014, in Protostars and Planets VI, ed. H. Beuther, R. S. Klessen, C. P. Dullemond, \& T. Henning, 835

Vastel, C., Phillips, T. G., \& Yoshida, H. 2004, ApJ, 606, L127

Visser, R., Doty, S. D., \& van Dishoeck, E. F. 2011, A\&A, 534, A132

Visser, R., van Dishoeck, E. F., \& Black, J. H. 2009, A\&A, 503, 323

Wakelam, V., Loison, J. C., Herbst, E., et al. 2015, ApJS, 217, 20

Wakelam, V., Loison, J. C., Mereau, R., \& Ruaud, M. 2017, Molecular Astrophysics, 6, 22

Ward-Thompson, D., André, P., Crutcher, R., et al. 2007, in Protostars and Planets V, ed. B. Reipurth, D. Jewitt, \& K. Keil, 33

Wolcott-Green, J. \& Haiman, Z. 2011, MNRAS, 412, 2603

Young, C. H. \& Evans, Neal J., I. 2005, ApJ, 627, 293 
Table A.1. Reactions with custom barrier widths. Note that deuterated variants of the reactions have been excluded from the list, but share the same barrier widths.

\begin{tabular}{lll}
\hline \hline Reaction & Barrier width $(\AA)$ & Reference \\
\hline $\mathrm{O}+\mathrm{CO} \rightarrow \mathrm{CO}_{2}$ & 1.25 & a,b \\
$\mathrm{H}+\mathrm{CH}_{4} \rightarrow \mathrm{CH}_{3}+\mathrm{H}_{2}$ & 2.17 & a,b \\
$\mathrm{H}+\mathrm{CO} \rightarrow \mathrm{HCO}$ & 2.0 & b \\
$\mathrm{H}+\mathrm{H}_{2} \mathrm{CO} \rightarrow \mathrm{CH}_{2} \mathrm{OH}$ & 2.0 & b \\
$\mathrm{H}+\mathrm{H}_{2} \mathrm{CO} \rightarrow \mathrm{CH}_{3} \mathrm{O}$ & 2.0 & b \\
$\mathrm{H}+\mathrm{CH}_{3} \mathrm{OH} \rightarrow \mathrm{CH}_{2} \mathrm{OH}+\mathrm{H}_{2}$ & 2.0 & b \\
$\mathrm{H}+\mathrm{CH}_{3} \mathrm{OH} \rightarrow \mathrm{CH}_{3} \mathrm{O}+\mathrm{H}_{2}$ & 2.0 & b \\
\hline
\end{tabular}

References. (a) Garrod \& Pauly (2011); (b) Garrod (2013); (c) Aikawa et al. (2012)

\section{Appendix A: Surface reactions with custom barriers}

Appendix B: Comparison of $\mathrm{D} / \mathrm{H}$ ratios toward $\mathbf{0 . 2 2}$ $\mathbf{M}_{\odot}$ protostars

\section{Appendix C: Density structure of the cores at onset of collapse}

Figure C.1 shows the radial density profile for the 9 protostars studied in this work at the onset of collapse. The three isolated sink particles show a smooth Bonner-Ebert-like profile with $\rho \propto r^{-2}$. The clustered sources also resemble a BonnerEbert profile, however several sinks show indications of additional over-densities within 10,000 au, e.g., sink M048-C and sink M101-C. Overall, the profiles of the isolated cores also exhibit slightly less scatter around the medians.

\section{Appendix D: Lowering the ambient extinction $A_{\mathrm{v}}^{\mathbf{0}}$}

In the fiducial model, the ambient cloud is assumed to shield the cores with an extinction of 5 mag. To determine the impact of a lower ambient extinction, models with an ambient extinction of $\mathrm{A}_{\mathrm{v}}^{0}=2$ mag are presented here for 6 of the 9 protostars. Physical and chemical parameters are similar to the fiducial model, i.e., $T=10 \mathrm{~K}, n_{\text {tot }}=2 \times 10^{4} \mathrm{~cm}^{-3}$, and a duration of $3 \mathrm{Myr}$. In this case, the $\mathrm{D} / \mathrm{H}$ ratios of water in the hot corino are slightly higher, and a larger spread in $\mathrm{D} / \mathrm{H}$ ratios is observed. Figures D.1 and D.2 show the comparison between isolated and clustered protostars with similar final masses. No apparent correlation with the environment in present. 


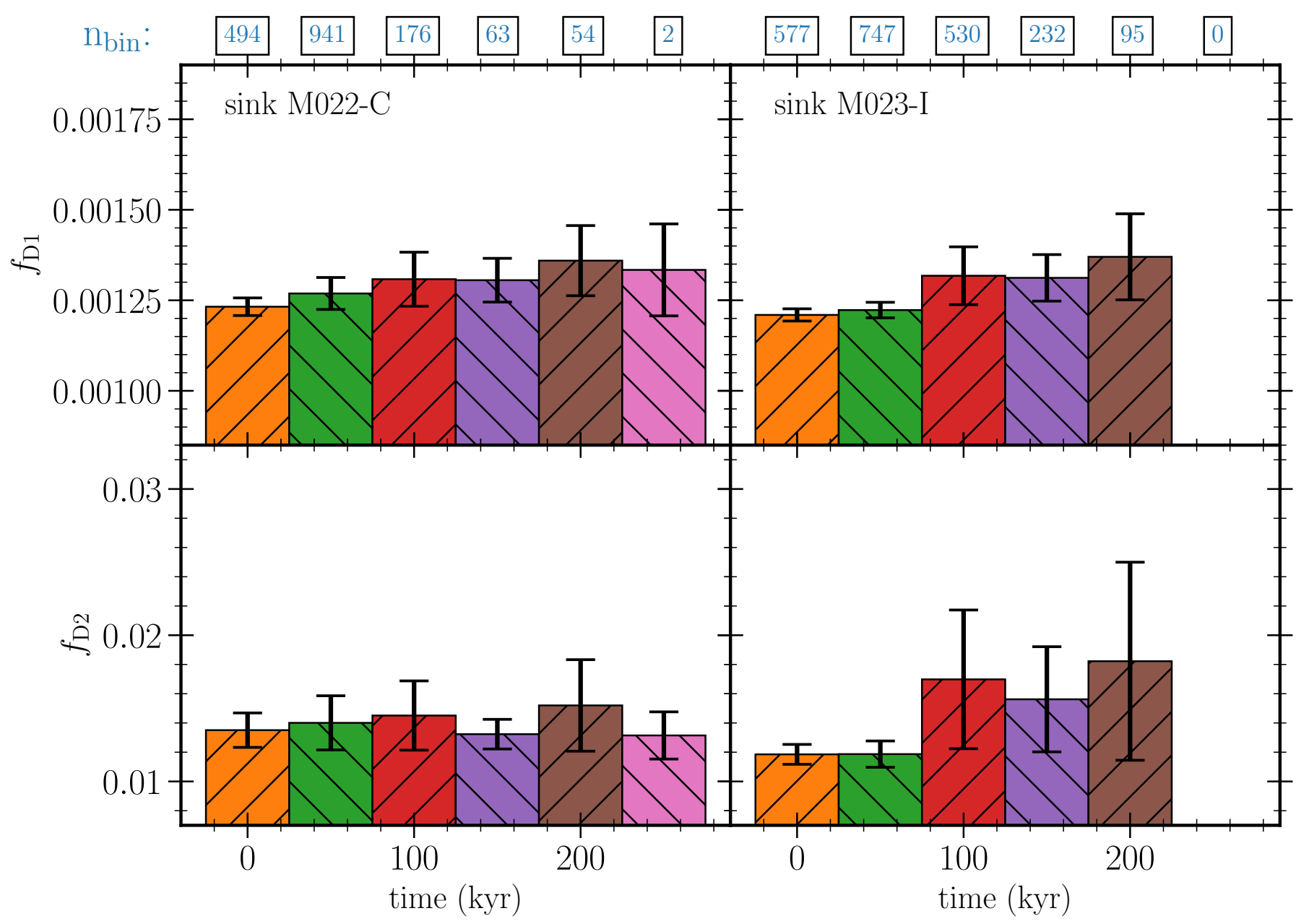

Fig. B.1. $\mathrm{HDO} / \mathrm{H}_{2} \mathrm{O}\left(f_{\mathrm{D} 1}\right)$ and $\mathrm{D}_{2} \mathrm{O} / \mathrm{HDO}\left(f_{\mathrm{D} 2}\right)$ ratios toward three protostars in the simulation, with similar final mass of $\sim 0.22 \mathrm{M}_{\odot}$. Tracer particles are binned according to the time at which they reach the hot corino, in bins with a width of 50 kyr. Time corresponds to the time after the onset of collapse, $t_{0}$. Each bar shows the median values of tracer particles accreted in the time interval, and the error shows the [15.9, 84.1] percentiles. The number of tracer particles within each bin is denoted in blue above the first row. 

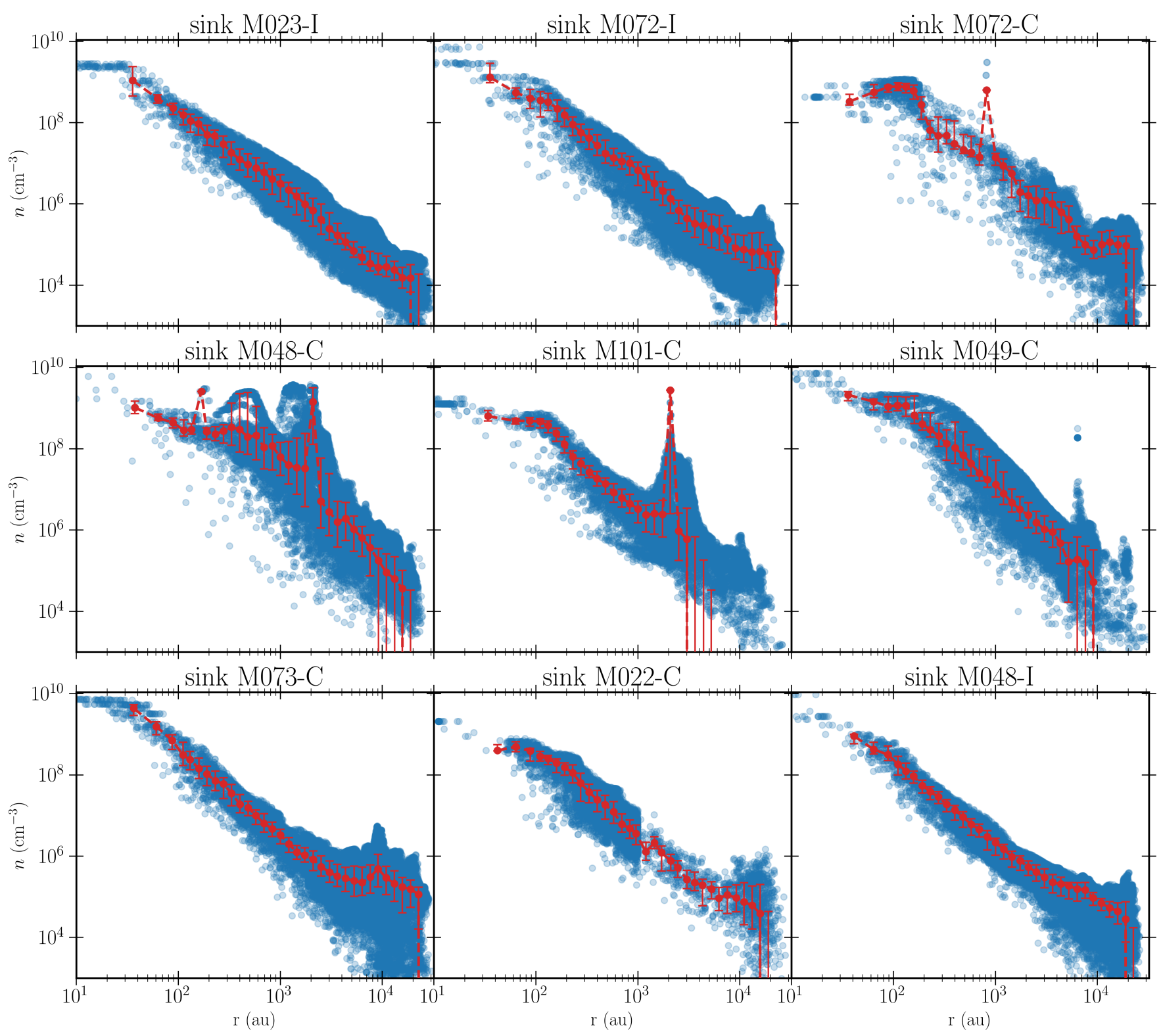

Fig. C.1. Radial density profile for 40,000 tracer particle for the sinks studied in this work. The red line shows the median in each radial bin, while the errorbars show the 25 and 75 percentiles for each bin. Blue circles indicates individual tracer particles. 


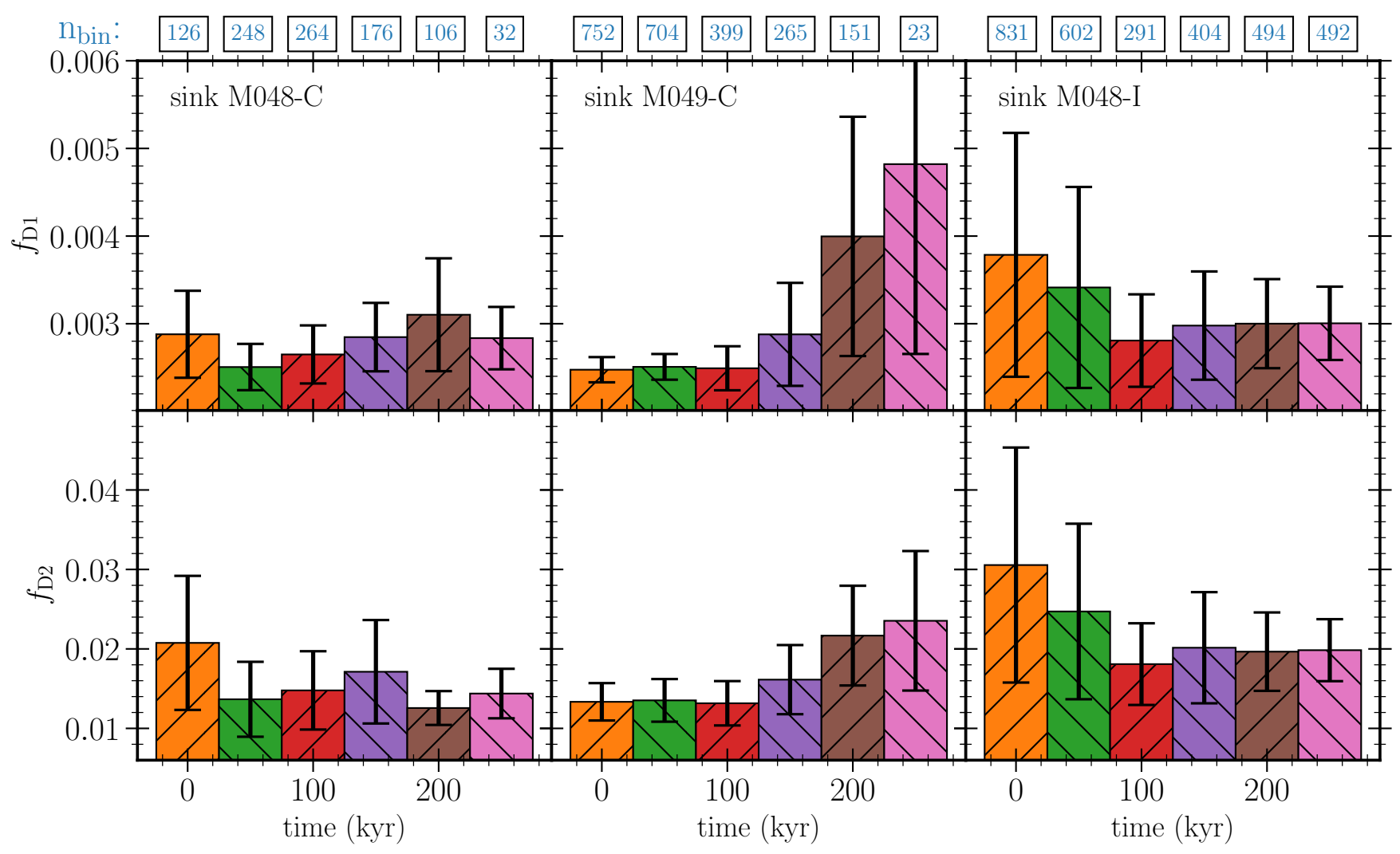

Fig. D.1. Similar to Fig. 10, with an ambient cloud extinction of 2 mag. $\mathrm{HDO} / \mathrm{H}_{2} \mathrm{O}\left(f_{\mathrm{D} 1}\right)$ and $\mathrm{D}_{2} \mathrm{O} / \mathrm{HDO}\left(f_{\mathrm{D} 2}\right)$ ratios toward three protostars in the simulation, with similar final mass of $\sim 0.5 \mathrm{M}_{\odot}$. Tracer particles are binned according to the time at which they reach the hot corino, in bins with a width of $50 \mathrm{kyr}$. Time corresponds to the time after the onset of collapse, $t_{0}$. The third protostar, sink M048-I, is classified as isolated since no protostar enter within 20,000 au during the simulation, while sink M048-C and sink M049-C are clustered. Each bar shows the median values of tracer particles accreted in the time interval, and the error shows the [15.9, 84.1] percentiles. The number of tracer particles within each bin is denoted in blue above the first row 


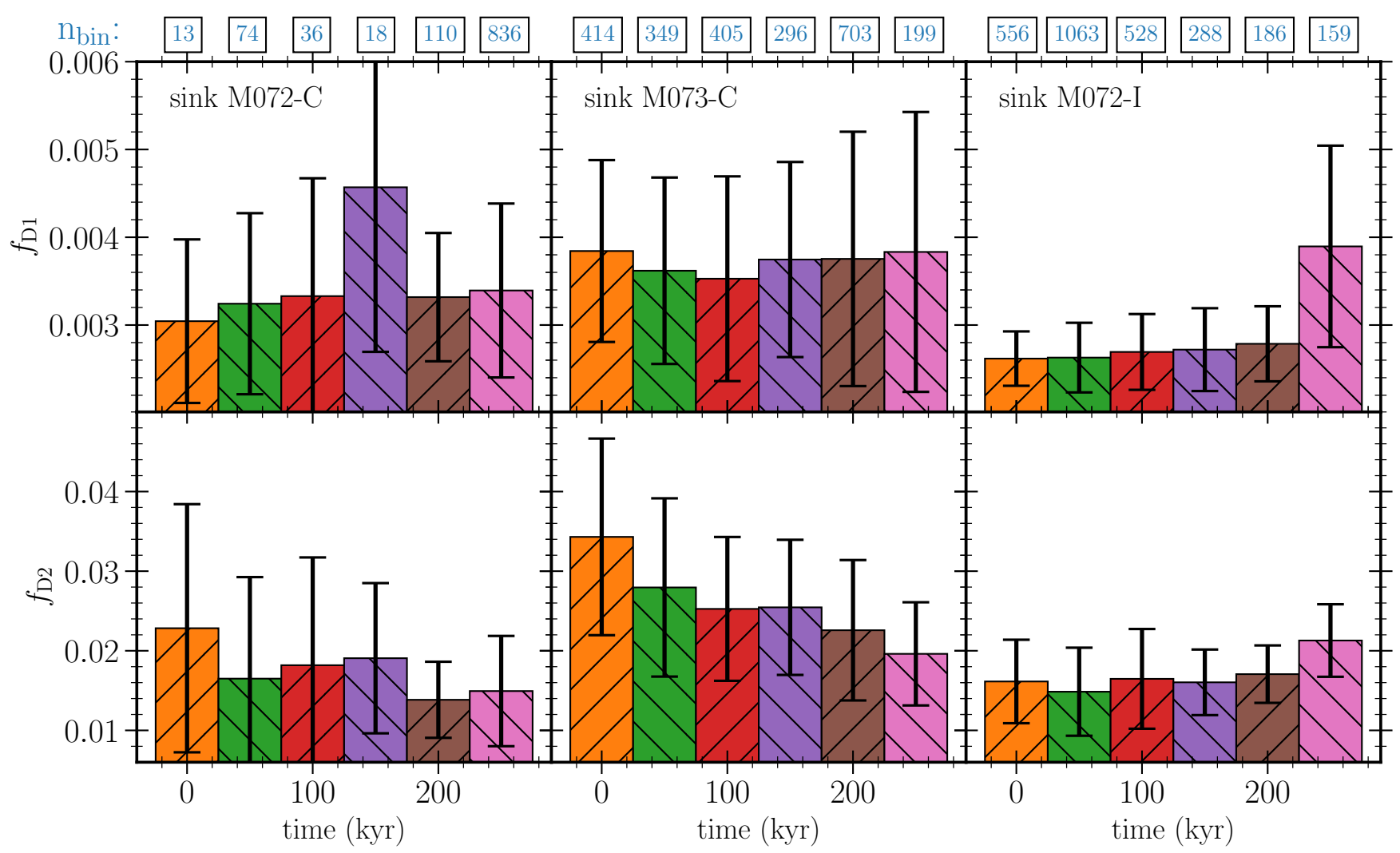

Fig. D.2. Similar to Fig. 11 , with an ambient cloud extinction of 2 mag. $\mathrm{HDO} / \mathrm{H}_{2} \mathrm{O}\left(f_{\mathrm{D} 1}\right)$ and $\mathrm{D}_{2} \mathrm{O} / \mathrm{HDO}\left(f_{\mathrm{D} 2}\right)$ ratios toward three protostars in the simulation, with similar final mass of $\sim 0.7 \mathrm{M}_{\odot}$. Tracer particles are binned according to the time at which they reach the hot corino, in bins with a width of $50 \mathrm{kyr}$. Time corresponds to the time after the onset of collapse, $t_{0}$. Each bar shows the median values of tracer particles accreted in the time interval, and the error shows the $[15.9,84.1]$ percentiles. The number of tracer particles within each bin is denoted in blue above the first row 